\title{
Coincidence Best Proximity Point Results in Branciari Metric Spaces with Applications
}

\author{
Naeem Saleem $\mathbb{D}^{1},{ }^{1}$ Manuel De la Sen $\mathbb{D}^{2},{ }^{2}$ and Sadia Farooq $\mathbb{D i}^{1}$ \\ ${ }^{1}$ Department of Mathematics, University of Management and Technology, Lahore, Pakistan \\ ${ }^{2}$ Institute of Research and Development of Processes, University of the Basque Country, Campus of Leioa (Bizkaia), P.O. Box 644, \\ Bilbao, Barrio Sarriena, 48940 Leioa, Spain
}

Correspondence should be addressed to Naeem Saleem; naeem.saleem2@gmail.com

Received 16 July 2020; Revised 30 August 2020; Accepted 27 November 2020; Published 14 December 2020

Academic Editor: Nawab Hussain

Copyright (C) 2020 Naeem Saleem et al. This is an open access article distributed under the Creative Commons Attribution License, which permits unrestricted use, distribution, and reproduction in any medium, provided the original work is properly cited.

This paper is aimed at studying the uniqueness of coincidence best proximity point for $\left(\vartheta, \alpha^{+}, \mathfrak{g}\right)$-proximal contractions in complete Branciari metric space. Throughout this article, discontinuity of the Branciari metric space is used and we obtained the desired results without assuming it as a continuous. Some examples are provided to validate the results proved herein. As an application, we derive the best proximity point results in the setup of complete Branciari metric space endowed with graph. Further, our results extend and generalize the existing ones in literature.

\section{Introduction and Preliminaries}

Let $\mathfrak{F}: \mathscr{X} \longrightarrow \mathscr{X}$ be a mapping, where $\mathscr{X}$ be any nonempty set. "An element $q^{*} \in \mathscr{X}$ is a fixed point of $\mathfrak{F}$ if $q^{*}$ satisfies the equation $\mathfrak{F} q^{*}=q^{*}$ (known as a fixed point equation) or $\mathfrak{D}\left(q^{*}, \mathfrak{\Im} q^{*}\right)=0$." A collection of all "fixed points" of $\mathfrak{\Im}$ will be represented as $\mathfrak{F}(\mathscr{X})$, that is,

$$
\mathfrak{F}(\mathscr{X})=\left\{q^{*} \in \mathscr{X}: \mathfrak{D}\left(q^{*}, \mathfrak{\mho} q^{*}\right)=0\right\} .
$$

In this direction, Banach [1] gives the existence and uniqueness of the "fixed point" of the self mapping $\mathfrak{F}$, if mapping $\mathfrak{F}$ is a contraction and $(\mathscr{X}, \mathfrak{D})$ is a complete, but it becomes more interesting, if $\mathfrak{F}$ is a nonself mapping then it is not necessary that the operator equation $\mathfrak{F} q^{*}=q^{*}$ has a solution. In this situation, we can find a point $q^{*} \in \mathscr{X}$ which is closest to $\mathfrak{F} q^{*}$ and we have the following minimization/optimization problem

$$
\min _{q \in \mathscr{X}} \mathfrak{d}(q, \mathfrak{F} q)
$$

Now, consider $\mathscr{X}=(\mathscr{X}, \mathfrak{D})$ be a metric space, $\mathscr{Q}$ and $\mathscr{P}$ are nonempty subsets of $\mathscr{X}$, and consider a mapping $\mathfrak{F}: \mathscr{Q} \longrightarrow$ $\mathscr{P}$, we can find a point $q^{*}$ in $\mathscr{Q}$ such that $\mathfrak{d}\left(q^{*}, \mathfrak{F} q^{*}\right)$ is mini- mum. In other words, we have to minimize $\mathfrak{d}\left(q^{*}, \mathfrak{F} q^{*}\right)$ for all $q^{*}$ in $\mathbb{Q}$ and $\mathfrak{F}^{*}$ in $\mathscr{P}$. It is important to see that the $\min _{q \in \mathscr{Q}} \mathfrak{d}(q, \mathfrak{F} q)=\mathfrak{d}(\mathscr{Q}, \mathscr{P})$, where $\mathfrak{d}(\mathscr{Q}, \mathscr{P})=\inf \{\mathfrak{d}(q, p): q$ $\in \mathscr{Q}, p \in \mathscr{P}\}$ which cannot be further reduced. If such point $q^{*}$ in $\mathbb{Q}$ exists then $q^{*}$ is called an "approximate fixed point" of $\mathfrak{F}[2]$.

Later, several authors studied the results dealing with "approximate fixed points" in different spaces (for detail, see [3-14]).

The best proximity point of the mapping $\mathfrak{F}: \mathscr{Q} \longrightarrow \mathscr{P}$ is actually "a point $q^{*} \in \mathscr{X}$ such that $\mathfrak{d}\left(q^{*}, \mathfrak{F} q^{*}\right)=\mathfrak{d}(\mathscr{Q}, \mathscr{P})$." Note that if $\mathscr{Q} \cap \mathscr{P} \neq \phi$ then $\mathfrak{D}(\mathscr{Q}, \mathscr{P})=0$; in this case, every "approximate fixed point" becomes "fixed point" of the mapping $\mathfrak{F}$. From this perspective, we can say that "the best proximity point results" are natural generalization of "fixed point results."

The concept of "coincidence best proximity point" was introduced in [5] for a pair of mappings in metric space. "A point $q^{*} \in \mathbb{Q}$ is called the coincidence best proximity point of a pair of mappings $\mathfrak{F}: \mathbb{Q} \longrightarrow \mathscr{P}$ and $\mathfrak{g}: \mathbb{Q} \longrightarrow \mathbb{Q}$ if $\mathfrak{d}\left(\mathfrak{g} q^{*}, \mathfrak{F}\right.$ $\left.q^{*}\right)=\mathfrak{d}(\mathscr{Q}, \mathscr{P})$." We denote the set of all "coincidence best proximity points" of a pair of mappings $\mathfrak{F}$ and $\mathfrak{g}$ by $\mathfrak{F} \mathfrak{g}(\mathbb{Q})$, that is,

$$
\mathfrak{F} \mathfrak{g}(\mathscr{Q})=\left\{q^{*} \in \mathscr{Q}: \mathfrak{d}\left(\mathfrak{g} q^{*}, \mathfrak{F} q^{*}\right)=\mathfrak{d}(\mathscr{Q}, \mathscr{P})\right\}
$$


This is an extension of a "best proximity point problem." If $\mathfrak{g}$ is an identity mapping on $\mathbb{Q}$ then a "coincidence best proximity point" will reduce to a "best proximity point" of mapping $\mathfrak{F}$.

Recently, Branciari [15] defined a Branciari (by some author generalized/rectangular) metric space. Branciari metric space generalizes the deterministic metric space in a natural way. Few examples are also provided to show that a Branciari metric space is not a metric space.

Definition 1 (see [15]). Let $(\mathscr{X}, \mathfrak{d})$ be a nonempty set. Any mapping $\mathfrak{d}: \mathscr{X} \times \mathscr{X} \longrightarrow[0, \infty)$ is a Branciari metric on $\mathscr{X}$, if for all $q, p \in \mathscr{X}, u, v \in \mathscr{X} \backslash\{q, p\}$, and the following conditions are satisfied:

(1) $\mathfrak{d}(q, p)=0$ if and only if $q=p$

(2) $\mathfrak{d}(q, p)=\mathfrak{d}(p, q)$

(3) $\mathfrak{d}(q, p) \leq \mathfrak{d}(q, u)+\mathfrak{d}(u, v)+\mathfrak{d}(v, p)$

Then, the pair $(\mathscr{X}, \mathfrak{d})$ is called a Branciari metric space.

Definition 2 (see [15]). Let $(\mathscr{X}, \mathfrak{d})$ be a Branciari metric space. Then, a sequence $\left\{q_{n}\right\}$ in $(\mathscr{X}, \mathfrak{d})$ is as follows:

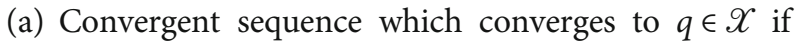
and only if $q_{n} \longrightarrow q$ as $n \longrightarrow \infty$. In this case, we can write

$$
\lim _{n \rightarrow \infty} q_{n}=q
$$

(b) Cauchy sequence if and only if $\mathfrak{d}\left(q_{n}, q_{m}\right) \longrightarrow 0$ as $n$, $m \longrightarrow \infty$

(c) A Branciari metric space $(\mathscr{X}, \mathfrak{d})$ is complete if every Cauchy sequence in $\mathscr{X}$ converges to some element in $\mathscr{X}$.

Lemma 3 (see [16]). Let $(\mathscr{X}, \mathfrak{d})$ be a Branciari metric space and $\left\{q_{n}\right\}$ be a Cauchy sequence in $\mathscr{X}$ such that $q_{m} \neq q_{n}$ whenever $m \neq n$. Then, the sequence $\left\{q_{n}\right\}$ can converge to utmost one point.

In a Branciari metric space, if a sequence is both Cauchy and convergent, then pathologies provided in an example [17] cannot happen, as shown in the following Lemma.

Lemma 4 (see [18]). Suppose that $\left\{q_{n}\right\}$ is a Cauchy sequence in a Branciari metric space $(\mathscr{X}, \mathfrak{d})$ with $\lim _{n \longrightarrow \infty} \mathfrak{d}\left(q_{n}, q\right)=0$, where $q \in \mathscr{X}$. Then, $\lim _{n \rightarrow \infty} \mathfrak{d}\left(q_{n}, u\right)=\mathfrak{d}(q, u)$, for all $u \in \mathscr{X}$. In particular, the sequence $\left\{q_{n}\right\}$ cannot converge to $u$ if $u \neq q$.

Recently, Jleli and Samet [4] introduced the concept of $\vartheta$ -contraction and proved a fixed point result for such mappings in the setup of Branciari metric spaces.
Definition 5 (see [4]). Let $\Delta_{\vartheta}$ be the set of all functions $\vartheta$ : $(0$, $\infty) \longrightarrow(1, \infty)$ satisfying the following conditions:

$\left(\theta_{1}\right) \vartheta$ is increasing

$\left(\theta_{2}\right)$ for any sequence $\left\{\kappa_{n}\right\}$ in $(0, \infty), \lim _{n \longrightarrow \infty} \kappa_{n}=0^{+}$if and only if $\lim _{n \longrightarrow \infty} \vartheta\left(\kappa_{n}\right)=1$

$\left(\theta_{3}\right)$ there exist $Y \in(0,1)$ and $\ell \in(0, \infty]$ such that $\lim _{\kappa \rightarrow 0^{+}} \vartheta(\kappa)-1 / \kappa^{Y}=\ell$

Definition 6 (see [4]). Let $(\mathscr{X}, \mathfrak{d})$ be a complete Branciari metric space and $\vartheta \in \Delta_{\vartheta}$. A mapping $\mathfrak{F}: \mathscr{X} \longrightarrow \mathscr{X}$ is called $\vartheta$ -contraction if

$$
\vartheta(\mathfrak{d}(\mathfrak{F}(q), \mathfrak{F}(p))) \leq[\vartheta(\mathfrak{d}(q, p))]^{\varsigma},
$$

for any $q, p \in \mathscr{X}$, where $\mathfrak{d}(\mathfrak{F}(q), \mathfrak{F}(p))>0$ and $0 \leq \varsigma<1$.

Theorem 7 (see [4]). Let $(\mathscr{X}, \mathfrak{D})$ be a Branciari metric space and $\mathfrak{F}: \mathscr{X} \longrightarrow \mathscr{X}$ be a $\vartheta$-contraction. Then, $\mathfrak{F}$ has a unique fixed point in $\mathscr{X}$.

Following definitions are also needed in the sequel.

Definition 8 . Let $(\mathscr{X}, \mathfrak{d})$ be a Branciari metric space and $\mathcal{Q}$ and $\mathscr{P}$ be two nonempty subsets of $\mathscr{X}$. Define

$$
\begin{aligned}
\mathfrak{D}(\mathscr{Q}, \mathscr{P}) & =\inf \{\mathfrak{d}(q, p): q \in \mathbb{Q}, p \in \mathscr{P}\}, \\
\mathscr{Q}_{0} & =\{q \in \mathscr{Q}: \mathfrak{d}(q, p)=\mathfrak{d}(\mathscr{Q}, \mathscr{P}), \text { for some } p \in \mathscr{P}\}, \\
\mathscr{P}_{0} & =\{q \in \mathscr{P}: \mathfrak{d}(q, p)=\mathfrak{d}(\mathscr{Q}, \mathscr{P}), \text { for some } p \in \mathscr{Q}\} .
\end{aligned}
$$

Definition 9 (see [19]). Let $\mathbb{Q}_{0}$ be a nonempty subset of $\mathbb{Q}$ and $\mathscr{P}_{0}$ be a nonempty subset of $\mathscr{P}$; then, the pair $(\mathscr{Q}, \mathscr{P})$ satisfies the "weak $P$-property," if

$$
\left.\begin{array}{l}
\mathfrak{d}\left(q_{1}, p_{1}\right)=\mathfrak{d}(\mathscr{Q}, \mathscr{P}) \\
\mathfrak{d}\left(q_{2}, p_{2}\right)=\mathfrak{d}(\mathscr{Q}, \mathscr{P})
\end{array}\right\} \text { implies } \mathfrak{d}\left(q_{1}, q_{2}\right) \leq \mathfrak{d}\left(p_{1}, p_{2}\right),
$$

for all $q_{1}, q_{2} \in Q_{0}$ and $p_{1}, p_{2} \in \mathscr{P}_{0}$.

Definition 10. A mapping $\mathfrak{g}: \mathbb{Q} \longrightarrow \mathbb{Q}$ is called the following:

(1) [20] An “isometry," if

$$
\mathfrak{d}(\mathfrak{g} q, \mathfrak{g} p)=\mathfrak{d}(q, p)
$$

(2) [21] An "expansive mapping," if

$$
\mathfrak{d}(\mathfrak{g} q, \mathfrak{g} p) \geq \mathfrak{d}(q, p)
$$

for any $q, p \in Q$

Proposition 11 (see [22]). A self mapping $\mathfrak{g}: \mathbb{Q} \longrightarrow \mathbb{Q}$ is said to satisfy $\alpha_{R}$ property if there exist a mapping $\alpha: Q \times Q \mathbb{Q} \longrightarrow$ 
$-\infty, \infty)$ such that

$$
\alpha(\mathfrak{g} q, \mathfrak{g} p) \geq 0 \text { implies that } \alpha(q, p) \geq 0
$$

for all $q, p \in \mathbb{Q}$.

Definition 12 (see [3]). Let $(\mathscr{X}, \mathfrak{d})$ be a metric space, $\mathbb{Q}$ is a nonempty subset of , $\alpha: \mathbb{Q} \times \mathbb{Q} \longrightarrow-\infty, \infty)$, and $\mathfrak{F}: \mathbb{Q} \longrightarrow$ $\mathscr{P}$ is a mapping. Then, $\mathbb{Q}$ is said to be $\alpha$-regular, if $\left\{q_{n}\right\}$ is a sequence in $\mathbb{Q}$ such that $\alpha\left(q_{n}, q_{n+1}\right) \geq 0$ and $q_{n} \longrightarrow q \in \mathbb{Q}$ as $n \longrightarrow \infty$ then $\alpha\left(q_{n}, q\right) \geq 0$ for all $n \in N$.

Now we have the following definitions:

Definition 13. If $\alpha: \mathbb{Q} \times \mathbb{Q} \longrightarrow-\infty, \infty)$ then mapping $\mathfrak{F}: \mathbb{Q}$ $\longrightarrow \mathscr{P}$ is called "triangular proximal $\alpha^{+}$-admissible," if

$$
\left.\begin{array}{l}
\alpha(q, p) \geq 0 \\
\mathfrak{d}(u, \mathfrak{F} q)=\mathfrak{d}(\mathscr{Q}, \mathscr{P}) \\
\mathfrak{d}(v, \mathfrak{F} p)=\mathfrak{d}(\mathscr{Q}, \mathscr{P})
\end{array}\right\} \text { implies that } \alpha(u, v) \geq 0
$$

$$
\left.\begin{array}{l}
\alpha(w, z) \geq 0 \\
\alpha(z, r) \geq 0
\end{array}\right\} \text { implies that } \alpha(w, r) \geq 0
$$

for all $q, p, u, v, w, z, r \in \mathbb{Q}$.

Example 1. Consider $\mathscr{X}=\mathscr{E}$ with the usual metric $\mathfrak{D}: \mathscr{X} \times$ $\mathscr{X} \longrightarrow \mathscr{E}$ defined by $\mathfrak{d}(q, p)=|q-p|$. Let $\mathbb{Q}$ and $\mathscr{P}$ be subsets of $\mathscr{X}$ defined by

$$
\begin{aligned}
Q & =\left\{\frac{1}{6}, \frac{3}{6}, \frac{5}{6}, \frac{7}{6}, \frac{9}{6}, \frac{11}{6}, \frac{13}{6}\right\}, \\
\mathscr{P} & =\left\{0, \frac{2}{6}, \frac{4}{6}, \frac{6}{6}\right\} .
\end{aligned}
$$

It is easy to see that $\mathfrak{d}(\mathscr{Q}, \mathscr{P})=1 / 6$. Let $\mathfrak{F}: \mathbb{Q} \longrightarrow \mathscr{P}$ be the mapping defined as

$$
\mathfrak{F}(q)=\left\{\begin{array}{l}
\frac{6}{6}, \text { if } q=\frac{3}{6} \\
0, \text { else }
\end{array}\right.
$$

Also, define $\alpha: \mathbb{Q} \times \mathbb{Q} \longrightarrow-\infty, \infty)$ by

$$
\alpha(q, p)=\left\{\begin{array}{l}
p-q \text { if } p>q \\
q-p \text { if } q \geq p
\end{array} \text { for all } q, p \in \mathbb{Q} .\right.
$$

$v=7 / 6$, and $u=1 / 6$ in $\mathbb{Q}$, we get

$$
\begin{aligned}
\alpha(q, p) & \geq 0, \\
\mathfrak{D}(u, \mathfrak{\mho} q) & =\mathfrak{d}(\mathscr{Q}, \mathscr{P}), \\
\mathfrak{d}(v, \mathfrak{\mho} p) & =\mathfrak{d}(\mathscr{Q}, \mathscr{P}),
\end{aligned}
$$

which implies that

$$
\alpha(u, v) \geq 0
$$

If we take $w, z, \kappa \in \mathbb{Q}$, we get

$$
\left.\begin{array}{l}
\alpha(w, z) \geq 0 \\
\alpha(z, \kappa) \geq 0
\end{array}\right\} \text { implies } \alpha(w, \kappa) \geq 0
$$

Case 2. If we take $q \in\{1 / 6,5 / 6,7 / 6,9 / 6,11 / 6,13 / 6\}, p=3 / 6$, $v=5 / 6$, and $u=1 / 6$ in $Q$, we get

$$
\begin{aligned}
\alpha(q, p) & \geq 0, \\
\mathfrak{d}(u, \mathfrak{J} q) & =\mathfrak{d}(\mathfrak{Q}, \mathscr{P}), \\
\mathfrak{d}(v, \mathfrak{J} p) & =\mathfrak{d}(\mathfrak{Q}, \mathscr{P}),
\end{aligned}
$$

which implies that

$$
\alpha(u, v) \geq 0
$$

If we take $w, z, \kappa \in Q$, we get

$$
\left.\begin{array}{l}
\alpha(w, z) \geq 0 \\
\alpha(z, \kappa) \geq 0
\end{array}\right\} \text { implies } \alpha(w, \kappa) \geq 0
$$

Definition 14. If $\mathfrak{F}: \mathbb{Q} \longrightarrow \mathscr{P}$ and $\mathfrak{g}: \mathbb{Q} \longrightarrow \mathbb{Q}$ then pair of mappings $(\mathfrak{F}, \mathfrak{g})$ satisfies

(1) $\left(\vartheta, \alpha^{+}, \mathfrak{g}\right)$-proximal contraction, if

$$
\begin{gathered}
\left.\begin{array}{c}
\alpha(q, p) \geq 0 \\
\mathfrak{d}(\mathfrak{g} u, \mathfrak{F} q)=\mathfrak{d}(\mathfrak{Q}, \mathscr{P}) \\
\mathfrak{d}(\mathfrak{g} v, \mathfrak{F} p)=\mathfrak{d}(\mathfrak{Q}, \mathscr{P})
\end{array}\right\} \text { implies } \\
\alpha(q, p)+\mathfrak{\vartheta}[\mathfrak{d}(\mathfrak{J} q, \mathfrak{F} p)] \leq[\vartheta(\mathfrak{d}(q, p))]^{\varsigma_{1}}[\vartheta(\mathfrak{d}(\mathfrak{g} u, \mathfrak{g} v))]^{\varsigma_{2}}
\end{gathered}
$$




$$
\begin{gathered}
\left.\begin{array}{c}
\alpha(q, p) \geq 0 \\
\mathfrak{d}(\mathfrak{g} u, \mathfrak{F} q)=\mathfrak{d}(\mathfrak{Q}, \mathscr{P}) \\
\mathfrak{d}(\mathfrak{g} v, \mathfrak{F} p)=\mathfrak{d}(\mathscr{Q}, \mathscr{P})
\end{array}\right\} \text { implies } \\
\alpha(q, p)+\mathfrak{\vartheta}[\mathfrak{d}(\mathfrak{g} u, \mathfrak{g} v)] \leq[\mathfrak{V}(\mathfrak{d}(q, p))]^{\varsigma_{1}}[\mathfrak{\vartheta}(\mathfrak{d}(u, v))]^{\varsigma_{2}},
\end{gathered}
$$

where , $p, u, v \in Q, \vartheta \in \Delta_{\vartheta}$, and $\varsigma_{1}, \varsigma_{2} \geq 0$ with $0 \leq \varsigma_{1}+\varsigma_{2}<1$

Definition 15. If $\mathfrak{F}: \mathbb{Q} \longrightarrow \mathscr{P}$ and $\mathfrak{g}: \mathbb{Q} \longrightarrow \mathbb{Q}$ then pair of mappings $(\mathfrak{F}, \mathfrak{g})$ satisfies

(1) $(\vartheta, \mathfrak{g})$-proximal contraction, if

$\left.\begin{array}{l}\mathfrak{d}(\mathfrak{g} u, \mathfrak{F} q)=\mathfrak{d}(\mathscr{Q}, \mathscr{P}) \\ \mathfrak{d}(\mathfrak{g} v, \mathfrak{F} p)=\mathfrak{d}(\mathscr{Q}, \mathscr{P})\end{array}\right\}$ implies $\mathfrak{\vartheta}[\mathfrak{d}(\mathfrak{F} q, \mathfrak{F} p)] \leq[\mathfrak{\vartheta}(\mathfrak{d}(q, p))]^{\varsigma_{1}}[\mathfrak{\vartheta}(\mathfrak{d}(\mathfrak{g} u, \mathfrak{g} v))]^{\varsigma_{2}}$

(2) “( $\vartheta, \mathfrak{g})$-generalized proximal contraction,” if

$\left.\begin{array}{l}\mathfrak{D}(\mathfrak{g} u, \mathfrak{F} q)=\mathfrak{d}(\mathscr{Q}, \mathscr{P}) \\ \mathfrak{D}(\mathfrak{g} v, \mathfrak{F} p)=\mathfrak{d}(\mathscr{Q}, \mathscr{P})\end{array}\right\}$ implies $\mathfrak{\vartheta}[\mathfrak{d}(\mathfrak{g} u, \mathfrak{g} \mathfrak{y})] \leq[\vartheta(\mathfrak{d}(q, p))]^{\varsigma_{1}}[\vartheta(\mathfrak{d}(u, v))]^{\varsigma_{2}}$,

where , $p, u, v \in Q, \vartheta \in \Delta_{\vartheta}$, and $\varsigma_{1}, \varsigma_{2} \geq 0$ with $0 \leq \varsigma_{1}+\varsigma_{2}<1$

Definition 16. Let $\mathfrak{F}: \mathbb{Q} \longrightarrow \mathscr{P}$ be a mapping satisfying

(1) $\left(\vartheta, \alpha^{+}\right)$-proximal contraction, if

$$
\begin{aligned}
& \left.\begin{array}{l}
\alpha(q, p) \geq 0 \\
\mathfrak{D}(u, \mathfrak{F} q)=\mathfrak{d}(\mathscr{Q}, \mathscr{P}) \\
\mathfrak{D}(v, \mathfrak{F} p)=\mathfrak{d}(\mathfrak{Q}, \mathscr{P})
\end{array}\right\} \text { implies } \\
& \alpha(q, p)+\vartheta[\mathfrak{d}(\mathfrak{F} q, \mathfrak{F} p)] \leq[\mathfrak{\vartheta}(\mathfrak{d}(q, p))]^{\varsigma_{1}}[\mathcal{V}(\mathfrak{d}(u, v))]^{\varsigma_{2}}
\end{aligned}
$$

(2) $\left(\vartheta, \alpha^{+}\right)$-generalized proximal contraction, if

$$
\begin{gathered}
\left.\begin{array}{c}
\alpha(q, p) \geq 0 \\
\mathfrak{d}(u, \mathfrak{J} q)=\mathfrak{d}(\mathscr{Q}, \mathscr{P}) \\
\mathfrak{d}(v, \mathfrak{F} p)=\mathfrak{d}(\mathscr{Q}, \mathscr{P})
\end{array}\right\} \text { implies } \\
\alpha(q, p)+\mathfrak{\vartheta}[\mathfrak{d}(u, v)] \leq[\vartheta(\mathfrak{d}(q, p))]^{\varsigma_{1}}[\vartheta(\mathfrak{D}(u, v))]^{\varsigma_{2}},
\end{gathered}
$$

where $q, p, u, v \in Q, \vartheta \in \Delta_{\vartheta}$, and $\varsigma_{1}, \varsigma_{2} \geq 0$ with $0 \leq \varsigma_{1}+\varsigma_{2}<1$.

Definition 17. Let $\mathfrak{F}: \mathscr{Q} \longrightarrow \mathscr{P}$ be a mapping satisfying

(1) ' 9 -proximal contraction, if

$$
\left.\begin{array}{l}
\mathfrak{d}(u, \mathfrak{F} q)=\mathfrak{d}(\mathscr{Q}, \mathscr{P}) \\
\mathfrak{d}(v, \mathfrak{F} p)=\mathfrak{d}(\mathscr{Q}, \mathscr{P})
\end{array}\right\} \text { implies } \mathfrak{\vartheta}[\mathfrak{d}(\mathfrak{\mho} q, \mathfrak{\mho} p)] \leq[\mathfrak{\vartheta}(\mathfrak{d}(q, p))]^{\varsigma_{1}}[\vartheta(\mathfrak{d}(u, v))]^{\varsigma_{2}}
$$

(2) $\vartheta$-generalized proximal contraction, if

$$
\left.\begin{array}{l}
\mathfrak{d}(u, \mathfrak{\Im} q)=\mathfrak{d}(\mathscr{Q}, \mathscr{P}) \\
\mathfrak{d}(v, \mathfrak{F} p)=\mathfrak{d}(\mathscr{Q}, \mathscr{P})
\end{array}\right\} \text { implies }[\vartheta(\mathfrak{d}(u, v))]^{1-\varsigma_{2}} \leq[\vartheta(\mathfrak{d}(q, p))]^{\varsigma_{1}},
$$

where, $p, u, v \in \mathbb{Q}, \vartheta \in \Delta_{\vartheta}$, and $\varsigma_{1}, \varsigma_{2} \geq 0$ with $0 \leq \varsigma_{1}+\varsigma_{2}<1$.

Remark 18. By taking $\mathfrak{g}=I_{\mathscr{Q}}$ (identity, mapping over $Q$ ) then every $\left(\vartheta, \alpha^{+}, \mathfrak{g}\right)$-proximal contraction will reduce to $\left(\vartheta, \alpha^{+}\right)$ -proximal contraction and $\left(\vartheta, \alpha^{+}, \mathfrak{g}\right)$-generalized proximal contraction will reduce to $\left(\vartheta, \alpha^{+}\right)$-generalized proximal contraction.

Remark 19. By taking $\alpha(q, p)=0$, every $\left(\vartheta, \alpha^{+}, \mathfrak{g}\right)$-proximal contraction will reduce to $(\vartheta, \mathfrak{g})$-proximal contraction and $\left(\vartheta, \alpha^{+}, \mathfrak{g}\right)$-generalized proximal contraction will reduce to $(\vartheta, \mathfrak{g})$-generalized proximal contraction.

Remark 20. By taking $\mathfrak{g}=I_{\mathscr{Q}}$ (identity, mapping over $\mathbb{Q}$ ) and $\alpha(q, p)=0$ then every $\left(\vartheta, \alpha^{+}, \mathfrak{g}\right)$-proximal contraction will reduce to $\vartheta$-proximal contraction and $\left(\vartheta, \alpha^{+}, \mathfrak{g}\right)$-generalized proximal contraction will reduce to $\vartheta$-generalized proximal contraction.

Note that now and onwards in this article, we assumed that $\mathcal{Q}$ and $\mathscr{P}$ are nonempty, distinct, and disjoint subsets of a complete Branciari metric space $(\mathscr{X}, \mathfrak{d})$ and $\mathscr{X}=(\mathscr{X}, \mathfrak{d})$ will represent a "complete Branciari metric space" until otherwise it is stated. Also, note that if $(\mathscr{Q}, \mathscr{P})$ is nonempty, weakly compact, and convex pair in Banach space $\mathscr{X}$, then $Q_{0}$ and $\mathscr{P}_{0}$ are nonempty ([23]).

\section{Main Results}

Now we state and prove our main result which runs as follows.

Theorem 21. Let $\alpha: \mathbb{Q} \times \mathbb{Q} \longrightarrow-\infty, \infty),(\mathfrak{F}, \mathfrak{g})$ be a pair of mappings satisfying $\left(\vartheta, \alpha^{+}, \mathfrak{g}\right)$-proximal contraction, where $\mathfrak{F}: \mathbb{Q} \longrightarrow \mathscr{P}$ be a triangular proximal $\alpha^{+}$-admissible and $\mathfrak{g}: \mathbb{Q} \longrightarrow \mathbb{Q}$ be a one to one expansive mapping satisfying $\alpha_{R}$ property. Moreover, if $\mathbb{Q}_{0}$ is a nonempty $\alpha$-regular closed set in $\mathscr{X}, \mathfrak{F}\left(\mathscr{Q}_{0}\right) \subseteq \mathscr{P}_{0}, \mathscr{Q}_{0} \subseteq \mathfrak{g}\left(\mathscr{Q}_{0}\right)$, and $(\mathscr{Q}, \mathscr{P})$ satisfy the weak P-property. Then, there exists a coincidence best proximity point of the pair of mappings $(\mathfrak{F}, \mathfrak{g})$ provided that there exists $q_{0}, q_{1} \in \mathcal{Q}_{0}$ such that

$$
\mathfrak{d}\left(\mathfrak{g} q_{1}, \mathfrak{F} q_{0}\right)=\mathfrak{d}(\mathcal{Q}, \mathscr{P}) \text { and } \alpha\left(q_{0}, q_{1}\right) \geq 0
$$


Moreover, if $\alpha(q, p) \geq 0$, for every $q, p \in \mathbb{Q}$ such that $\mathfrak{D}$ $(\mathfrak{g} q, \mathfrak{F} q)=\mathfrak{d}(\mathscr{Q}, \mathscr{P})=\mathfrak{d}(\mathfrak{g} p, \mathfrak{F} p)$ then $q^{*}$ is the unique coincidence best proximity point of the pair of mappings $(\mathfrak{F}, \mathfrak{g})$.

Proof. Let $q_{0}, q_{1} \in \mathscr{Q}_{0}$ such that $\mathfrak{d}\left(\mathfrak{g} q_{1}, \mathfrak{\mho} q_{0}\right)=\mathfrak{d}(\mathscr{Q}, \mathscr{P})$ and $\alpha$ $\left(q_{0}, q_{1}\right) \geq 0$. As $\mathfrak{F} q_{0} \in \mathfrak{F}\left(\mathscr{Q}_{0}\right) \subseteq \mathscr{P}_{0}$ and $\mathfrak{g} q_{1} \in \mathscr{Q}_{0} \subseteq \mathfrak{g}\left(\mathbb{Q}_{0}\right)$, there exists $q_{2} \in \mathscr{Q}_{0}$ such that $\mathfrak{d}\left(\mathfrak{g} q_{2}, \mathfrak{F} q_{1}\right)=\mathfrak{d}(\mathscr{Q}, \mathscr{P})$. Since $\mathfrak{F}$ is a triangular proximal $\alpha^{+}$-admissible, we have $\alpha\left(\mathfrak{g} q_{1}, \mathfrak{g}\right.$ $\left.q_{2}\right) \geq 0$. Since $\mathfrak{g}$ satisfies $\alpha_{R}$ property, hence $\alpha\left(q_{1}, q_{2}\right) \geq 0$. Similarly, by $\mathfrak{F}\left(\mathscr{Q}_{0}\right) \subseteq \mathscr{P}_{0}$ and $Q_{0} \subseteq \mathfrak{g}\left(\mathscr{Q}_{0}\right)$, there exists a point $q_{3} \in \mathbb{Q}_{0}$ such that $\mathfrak{d}\left(\mathfrak{g} q_{3}, \mathfrak{\mho} q_{2}\right)=\mathfrak{d}(\mathscr{Q}, \mathscr{P})$. Since $\mathfrak{F}$ is a triangular proximal $\alpha^{+}$-admissible, this further implies that $\alpha\left(\mathfrak{g} q_{2}\right.$, $\left.\mathfrak{g} q_{3}\right) \geq 0$. Following the same arguments, we have $\alpha\left(q_{2}, q_{3}\right)$ $\geq 0$. Continuing this way, we can obtain a sequence $\left\{q_{n}\right\}$ in $Q_{0}$ such that

$$
\begin{aligned}
\mathfrak{D}\left(\mathfrak{g} q_{n}, \mathfrak{\mho} q_{n-1}\right) & =\mathfrak{d}(\mathscr{Q}, \mathscr{P})=\mathfrak{d}\left(\mathfrak{g} q_{n+1}, \mathfrak{F} q_{n}\right), \alpha\left(q_{n}, q_{n+1}\right) \\
& \geq 0, \text { for all } n \in \mathbb{N} \cup\{0\} .
\end{aligned}
$$

If $q_{n} \neq q_{n+1}$, for all $n \in \mathbb{N} \cup\{0\}$, the pair of mappings ( $\mathfrak{F}$, $\mathfrak{g})$ is $\left(\vartheta, \alpha^{+}, \mathfrak{g}\right)$-proximal contraction, and we have

$\alpha\left(q_{n}, q_{n-1}\right)+\vartheta\left(\mathfrak{d}\left(\widetilde{\mho} q_{n-1}, \mathfrak{\mho} q_{n}\right)\right) \leq\left[\vartheta\left(\mathfrak{d}\left(q_{n-1}, q_{n}\right)\right)\right]^{\mathcal{S}_{1}}\left[\vartheta\left(\mathfrak{d}\left(\mathfrak{g} q_{n+1}, \mathfrak{g} q_{n}\right)\right)\right]^{\varsigma_{2}}$. that

Since, $\mathfrak{F}$ is a triangular proximal $\alpha^{+}$-admissible, we obtain

$$
\vartheta\left(\mathfrak{D}\left(\mathfrak{\mho} q_{n-1}, \mathfrak{\mho} q_{n}\right)\right) \leq\left[\vartheta\left(\mathfrak{d}\left(q_{n-1}, q_{n}\right)\right)\right]^{\varsigma_{1}}\left[\vartheta\left(\mathfrak{D}\left(\mathfrak{g} q_{n+1}, \mathfrak{g} q_{n}\right)\right)\right]^{\varsigma_{2}} .
$$

Since pair $(\mathscr{Q}, \mathscr{P})$ satisfies the weak $P$-property and $\mathfrak{g}$ is one to one on $\mathbb{Q}_{0}$ then we have

$$
\begin{aligned}
\mathcal{\vartheta}\left(\mathfrak{d}\left(\mathfrak{g} q_{n}, \mathfrak{g} q_{n+1}\right)\right) & \leq \mathfrak{\vartheta}\left(\mathfrak{d}\left(\mathfrak{\mho} q_{n-1}, \mathfrak{F} q_{n}\right)\right) \\
& \leq\left[\mathfrak{\vartheta}\left(\mathfrak{d}\left(q_{n-1}, q_{n}\right)\right)\right]^{\varsigma_{1}}\left[\vartheta\left(\mathfrak{d}\left(\mathfrak{g} q_{n+1}, \mathfrak{g} q_{n}\right)\right)\right]^{\varsigma_{2}},
\end{aligned}
$$

which further implies that

$$
\left[\vartheta\left(\mathfrak{d}\left(\mathfrak{g} q_{n}, \mathfrak{g} q_{n+1}\right)\right)\right]^{1-\varsigma_{2}} \leq\left[\mathfrak{V}\left(\mathfrak{d}\left(q_{n-1}, q_{n}\right)\right)\right]^{\varsigma_{1}},
$$

since $\mathfrak{g}$ is an expansive and $\vartheta$ is an increasing mapping; hence,

$\left[\mathfrak{\vartheta}\left(\mathfrak{d}\left(q_{n}, q_{n+1}\right)\right)\right]^{1-\varsigma_{2}} \leq\left[\mathfrak{\vartheta}\left(\mathfrak{d}\left(\mathfrak{g} q_{n}, \mathfrak{g} q_{n+1}\right)\right)\right]^{1-\varsigma_{2}} \leq\left[\mathfrak{\vartheta}\left(\mathfrak{d}\left(q_{n-1}, q_{n}\right)\right)\right]^{\varsigma_{1}}$.

After simplification, we have

$$
\vartheta\left(\mathfrak{d}\left(q_{n}, q_{n+1}\right)\right) \leq\left[\vartheta\left(\mathfrak{d}\left(q_{n-1}, q_{n}\right)\right)\right]^{\left(\varsigma_{1} / 1-\varsigma_{2}\right)} .
$$

Further, we can write as

$$
\left[\vartheta\left(\mathfrak{d}\left(q_{n}, q_{n+1}\right)\right)\right] \leq\left[\vartheta\left(\mathfrak{d}\left(q_{n-2}, q_{n-1}\right)\right)\right]^{\left(\varsigma_{1} / 1-\varsigma_{2}\right)^{2}} \leq\left[\vartheta\left(\mathfrak{d}\left(q_{0}, q_{1}\right)\right)\right]^{\left(\varsigma_{1} / 1-\varsigma_{2}\right)^{n}},
$$

which implies that

$$
\vartheta\left(\mathfrak{d}\left(q_{n}, q_{n+1}\right)\right) \leq\left[\vartheta\left(\mathfrak{d}\left(q_{0}, q_{1}\right)\right)\right]^{n^{n}}, \text { for all } n \in \mathbb{N},
$$

where $v=\varsigma_{1} / 1-\varsigma_{2}<1$. Taking limit $n \longrightarrow \infty$ in the above inequality, we have

$$
\lim _{n \longrightarrow \infty} \vartheta\left(\mathfrak{D}\left(q_{n}, q_{n+1}\right)\right)=1,
$$

which implies that

$$
\lim _{n \longrightarrow \infty} \mathfrak{d}\left(q_{n}, q_{n+1}\right)=0 .
$$

Since $\vartheta \in \Delta_{\vartheta}$ then there exist $0<Y<1$ and $0<\ell \leq \infty$, such that the following limit holds true:

$$
\lim _{n \longrightarrow \infty} \frac{\vartheta\left(\mathfrak{d}\left(q_{n}, q_{n+1}\right)\right)-1}{\left[\mathfrak{d}\left(q_{n}, q_{n+1}\right)\right]^{Y}}=\ell .
$$

Assume that $\ell<\infty$ and $C=\ell / 2$. Thus, there exist $n_{0} \in \mathbb{N}$, such that

$$
\left|\frac{\mathcal{Y}\left(\mathfrak{d}\left(q_{n}, q_{n+1}\right)\right)-1}{\left[\mathfrak{d}\left(q_{n}, q_{n+1}\right)\right]^{Y}}-\ell\right| \leq C, \text { for all } n \geq n_{0} .
$$

Hence, we have

$$
\frac{\vartheta\left(\mathfrak{d}\left(q_{n}, q_{n+1}\right)\right)-1}{\left[\mathfrak{d}\left(q_{n}, q_{n+1}\right)\right]^{Y}} \geq \ell-C=\frac{\ell}{2}=C, \text { for all } n \geq n_{0} .
$$

Further, we can write as

$$
n\left[\mathfrak{d}\left(q_{n}, q_{n+1}\right)\right]^{Y} \leq \omega D\left[\vartheta\left(\mathfrak{d}\left(q_{n}, q_{n+1}\right)\right)-1\right] \text {, for all } n \geq n_{0},
$$

where $\mathfrak{d}=1 / C$. If $\ell=\infty$ then there exists $n_{0} \in \mathbb{N}$, such that

$$
\frac{\mathcal{Y}\left(\mathfrak{d}\left(q_{n}, q_{n+1}\right)\right)-1}{\left[\mathfrak{d}\left(q_{n}, q_{n+1}\right)\right]^{Y}} \geq C, \text { for all } n \geq n_{0},
$$

which implies that

$$
n\left[\mathfrak{d}\left(q_{n}, q_{n+1}\right)\right]^{Y} \leq \omega D\left[\vartheta\left(\mathfrak{d}\left(q_{n}, q_{n+1}\right)\right)-1\right] \text {, for all } n \geq n_{0},
$$

where $D=1 / C$. Hence, in all cases, there exist $D>0$ and $n_{0}$ $\in \mathbb{N}$, such that

$$
n\left[\mathfrak{d}\left(q_{n}, q_{n+1}\right)\right]^{Y} \leq \omega D\left[\vartheta\left(\mathfrak{D}\left(q_{n}, q_{n+1}\right)\right)-1\right], \text { for all } n \geq n_{0} .
$$

From inequalities (39) and (48), we have

$$
n\left[\mathfrak{d}\left(q_{n}, q_{n+1}\right)\right]^{Y} \leq \omega D\left[\left(\vartheta\left(\mathfrak{D}\left(q_{0}, q_{1}\right)\right)\right)^{v^{n}}-1\right], \text { for all } n \geq n_{0} .
$$

Then, by taking limit as $n \longrightarrow \infty$ on both sides of the 
above inequality, we obtain

$$
n\left[\mathfrak{d}\left(q_{n}, q_{n+1}\right)\right]^{Y}=0 .
$$

Thus, there exists $n_{0} \in \mathbb{N}$ such that

$$
\mathfrak{d}\left(q_{n}, q_{n+1}\right) \leq \frac{1}{n^{1 / Y}} \text {, for all } n \geq n_{0} .
$$

Now suppose that $q_{n}=q_{m}$ for all $n, m \in \mathbb{N}$ and $n=m$. If $q_{n}$ $\neq q_{n+2}$, for all $n \in \mathbb{N} \cup\{0\}$ then

$$
\mathfrak{d}\left(\mathfrak{g} q_{n}, \mathfrak{F} q_{n-1}\right)=\mathfrak{d}\left(\mathfrak{g} q_{n+2}, \mathfrak{F} q_{n+1}\right)=\mathfrak{d}(\mathscr{Q}, \mathscr{P})
$$

Since $\mathfrak{F}$ is a triangular proximal $\alpha^{+}$-admissible $\alpha\left(q_{n-1}\right.$, $\left.q_{n}\right) \geq 0$ and $\alpha\left(q_{n}, q_{n+1}\right) \geq 0$, by (T2) of Definition 13, we have

$$
\alpha\left(q_{n-1}, q_{n+1}\right) \geq 0
$$

Again, since the pair of mappings $(\mathfrak{F}, \mathfrak{g})$ is $\left(\vartheta, \alpha^{+}, \mathfrak{g}\right)$ -proximal contraction, we have

$$
\begin{aligned}
\vartheta\left(\mathfrak{d}\left(\mathfrak{\mho} q_{n-1}, \mathfrak{J} q_{n+1}\right)\right) & \leq \alpha\left(q_{n-1}, q_{n+1}\right)+\vartheta\left(\mathfrak{d}\left(\mathfrak{\Im} q_{n-1}, \mathfrak{F} q_{n+1}\right)\right) \\
& \leq\left[\vartheta\left(\mathfrak{d}\left(q_{n-1}, q_{n+1}\right)\right)\right]^{\varsigma_{1}}\left[\vartheta\left(\mathfrak{d}\left(\mathfrak{g} q_{n+2}, \mathfrak{g} q_{n}\right)\right)\right]^{\varsigma_{2}}
\end{aligned}
$$

The pair $(\mathscr{Q}, \mathscr{P})$ satisfies the weak $P$-property; $\mathfrak{g}$ is one to one on $Q_{0}$, and $\vartheta$ is increasing; then,

$$
\vartheta\left(\mathfrak{d}\left(\mathfrak{g} q_{n}, \mathfrak{g} q_{n+2}\right)\right) \leq \mathfrak{\vartheta}\left(\mathfrak{d}\left(\mathfrak{F} q_{n-1}, \mathfrak{F} q_{n+1}\right)\right)
$$

Then, from inequalities (54) and (55), we have

$$
\mathfrak{\vartheta}\left(\mathfrak{d}\left(\mathfrak{g} q_{n}, \mathfrak{g} q_{n+2}\right)\right) \leq\left[\vartheta\left(\mathfrak{d}\left(q_{n-1}, q_{n+1}\right)\right)\right]^{\varsigma_{1}}\left[\vartheta\left(\mathfrak{d}\left(\mathfrak{g} q_{n+2}, \mathfrak{g} q_{n}\right)\right)\right]^{\varsigma_{2}},
$$

which further implies that

$$
\left[\mathfrak{\vartheta}\left(\mathfrak{d}\left(\mathfrak{g} q_{n+2}, \mathfrak{g} q_{n}\right)\right)\right]^{1-\varsigma_{2}} \leq\left[\vartheta\left(\mathfrak{d}\left(q_{n-1}, q_{n+1}\right)\right)\right]^{\varsigma_{1}}
$$

Since $\mathfrak{g}$ is an expansive mapping and $\vartheta$ is an increasing mapping, then we have

$\left[\vartheta\left(\mathfrak{d}\left(q_{n+2}, q_{n}\right)\right)\right]^{1-\varsigma_{2}} \leq\left[\vartheta\left(\mathfrak{d}\left(\mathfrak{g} q_{n+2}, \mathfrak{g} q_{n}\right)\right)\right]^{1-\varsigma_{2}} \leq\left[\vartheta\left(\mathfrak{d}\left(q_{n-1}, q_{n+1}\right)\right)\right]^{\varsigma_{1}}$.

After further simplifications, we have

$$
\begin{aligned}
\vartheta\left(\mathfrak{D}\left(q_{n+2}, q_{n}\right)\right) & \leq\left[\vartheta\left(\mathfrak{d}\left(q_{n-1}, q_{n+1}\right)\right)\right]^{\left(\varsigma_{1} / 1-\varsigma_{2}\right)} \\
& \leq\left[\vartheta\left(\mathfrak{d}\left(q_{n-2}, q_{n}\right)\right)\right]^{\left(\varsigma_{1} / 1-\varsigma_{2}\right)^{2}} \\
& \leq\left[\vartheta\left(\mathfrak{d}\left(q_{0}, q_{2}\right)\right)\right]^{\left(\varsigma_{1} / 1-\varsigma_{2}\right)^{n}}
\end{aligned}
$$

which implies that

$$
\vartheta\left(\mathfrak{d}\left(q_{n+2}, q_{n}\right)\right) \leq\left[\vartheta\left(\mathfrak{d}\left(q_{0}, q_{2}\right)\right)\right]^{\left(c_{1} / 1-\varsigma_{2}\right)^{n}}, \text { for all } n \in \mathbb{N},
$$

where $v=\varsigma_{1} / 1-\varsigma_{2}<1$. Taking the limit $n \longrightarrow \infty$ in above inequality, we have

$$
\lim _{n \rightarrow \infty} \vartheta\left(\mathfrak{d}\left(q_{n+2}, q_{n}\right)\right)=1
$$

Similarly, from condition $\left(\vartheta_{2}\right)$, we have

$$
\lim _{n \rightarrow \infty} \mathfrak{d}\left(q_{n}, q_{n+2}\right)=0
$$
that

Similarly, from condition $\left(\vartheta_{3}\right)$, there exists $n_{1} \in \mathbb{N}$, such

$$
\mathfrak{d}\left(q_{n}, q_{n+2}\right) \leq \frac{1}{n^{1 / Y}}, \text { for all } n \geq n_{1} .
$$

Now we have the following cases:

Case 1. If $m>2$ and $m$ is odd. Consider $m=2 \kappa+1, \kappa \geq 1$, using (51), and we obtain

$$
\begin{aligned}
\mathfrak{d}\left(q_{n}, q_{n+m}\right) \leq & \mathfrak{d}\left(q_{n}, q_{n+1}\right)+\mathfrak{d}\left(q_{n+1}, q_{n+2}\right)+\mathfrak{d}\left(q_{n+2}, q_{n+m}\right) \\
\leq & \mathfrak{d}\left(q_{n}, q_{n+1}\right)+\mathfrak{d}\left(q_{n+1}, q_{n+2}\right)+\mathfrak{d}\left(q_{n+2}, q_{n+3}\right) \\
& +\mathfrak{d}\left(q_{n+3}, q_{n+4}\right)+\mathfrak{d}\left(q_{n+4}, q_{n+m}\right) \\
\leq \leq & \mathfrak{d}\left(q_{n}, q_{n+1}\right)+\mathfrak{d}\left(q_{n+1}, q_{n+2}\right)+\mathfrak{d}\left(q_{n+2}, q_{n+3}\right)+\cdots \\
& +\mathfrak{d}\left(q_{n+m-1}, q_{n+m}\right) \leq \frac{1}{n^{1 / Y}}+\frac{1}{(n+1)^{1 / Y}} \\
& +\frac{1}{(n+2)^{1 / Y}}+\cdots+\frac{1}{(n+m-1)^{1 / Y}} \\
\mathfrak{d}\left(q_{n}, q_{n+m}\right) \leq & \sum_{i=n}^{\infty} \frac{1}{i^{1 / Y}},
\end{aligned}
$$

for all $m \geq n \geq N$, where $N=\max \left\{n_{0}, n_{1}\right\}$.

Case 2. If $m>2$ is even. Consider $m=2 \kappa, \kappa \geq 2$, using (51) and (63), and we obtain

$$
\begin{aligned}
\mathfrak{d}\left(q_{n}, q_{n+m}\right) \leq & \mathfrak{d}\left(q_{n}, q_{n+2}\right)+\mathfrak{d}\left(q_{n+2}, q_{n+3}\right)+\mathfrak{d}\left(q_{n+3}, q_{n+m}\right) \\
\leq & \mathfrak{d}\left(q_{n}, q_{n+2}\right)+\mathfrak{d}\left(q_{n+2}, q_{n+3}\right)+\mathfrak{d}\left(q_{n+3}, q_{n+4}\right) \\
& +\mathfrak{d}\left(q_{n+4}, q_{n+5}\right)+\mathfrak{d}\left(q_{n+5}, q_{n+m}\right) \\
\leq & \mathfrak{d}\left(q_{n}, q_{n+2}\right)+\mathfrak{d}\left(q_{n+2}, q_{n+3}\right)+\mathfrak{d}\left(q_{n+3}, q_{n+4}\right)+\cdots \\
& +\mathfrak{d}\left(q_{n+m-1}, q_{n+m}\right) \leq \frac{1}{n^{1 / Y}}+\frac{1}{(n+2)^{1 / Y}}+\cdots \\
& +\frac{1}{(n+m-1)^{1 / Y}} \leq \sum_{i=n}^{\infty} \frac{1}{i^{1 / Y}},
\end{aligned}
$$

for all $m \geq n \geq N$, where $N=\max \left\{n_{0}, n_{1}\right\}$. Thus, by combining all the cases, we have

$$
\mathfrak{d}\left(q_{n}, q_{n+m}\right) \leq \sum_{i=n}^{\infty} \frac{1}{i^{1 / Y}} \text {, for all } m \geq n \geq N,
$$

where $N=\max \left\{n_{0}, n_{1}\right\}$. Then, by $P$-series test, $\sum_{i=n}^{\infty}\left(1 / i^{1 / Y}\right)$ 
converges as $1 / Y>1$. We deduce that $\left\{q_{n}\right\}$ is a Cauchy sequence in $\mathbb{Q}_{0} \subseteq Q \mathcal{Q} \subset \mathscr{X}$. By the completeness of space $\mathscr{X}$ and $\mathscr{Q}_{0}$ is closed, there exists $q^{*} \in \mathscr{Q}_{0}$ such that $q_{n} \longrightarrow q^{*} \in$ $\mathbb{Q}_{0}$. Since $\widehat{Q}_{0} \subseteq \mathfrak{g}\left(\mathscr{Q}_{0}\right)$, we have $q^{*}=\mathfrak{g} q^{*}$. Since $\mathbb{Q}_{0}$ is $\alpha$-regular then $\alpha\left(q_{n}, q^{*}\right) \geq 0$. Since $q^{*} \in \mathbb{Q}_{0}, \mathfrak{F}\left(\mathcal{Q}_{0}\right) \subseteq \mathscr{P}_{0}$ then $\mathfrak{F} q^{*}$ $\in \mathscr{P}_{0}$; thus, there exists a point $z \in \mathscr{Q}_{0}$ such that $z=q^{*}$ and

$$
\mathfrak{d}\left(\mathfrak{g} z, \mathfrak{F} q^{*}\right)=\mathfrak{d}(\mathscr{Q}, \mathscr{P})
$$

Since the pair of mappings $(\mathfrak{F}, \mathfrak{g})$ is $\left(\vartheta, \alpha^{+}, \mathfrak{g}\right)$-proximal contraction and by using weak $P$-property, we obtain

$$
\begin{aligned}
\vartheta\left(\mathfrak{d}\left(\mathfrak{g} q_{n+1}, \mathfrak{g} z\right)\right) & \leq \mathfrak{\vartheta}\left(\mathfrak{d}\left(\mathfrak{\mho} q_{n}, \mathfrak{F} q^{*}\right)\right) \leq \alpha\left(q_{n}, q^{*}\right)+\vartheta\left(\mathfrak{d}\left(\mathfrak{\mho} q_{n}, \mathfrak{F} q^{*}\right)\right) \\
& \leq\left[\vartheta\left(\mathfrak{D}\left(q_{n}, q^{*}\right)\right)\right]^{\varsigma_{1}}\left[\vartheta\left(\mathfrak{d}\left(\mathfrak{g} q_{n+1}, \mathfrak{g} z\right)\right)\right]^{\varsigma_{2}} .
\end{aligned}
$$

Further, the above inequality can be written as follows:

$$
\left[\vartheta\left(\mathfrak{d}\left(\mathfrak{g} q_{n+1}, \mathfrak{g} z\right)\right)\right]^{1-\varsigma_{2}} \leq\left[\vartheta\left(\mathfrak{d}\left(q_{n}, q^{*}\right)\right)\right]^{\varsigma_{1}} .
$$

Since $\mathfrak{g}$ is an expansive mapping, we have

$$
\begin{aligned}
{\left[\vartheta\left(\mathfrak{D}\left(q_{n+1}, z\right)\right)\right]^{1-\varsigma_{2}} } & \leq\left[\vartheta\left(\mathfrak{d}\left(\mathfrak{g} q_{n+1}, \mathfrak{g} z\right)\right)\right]^{1-\varsigma_{2}} \\
& \leq\left[\vartheta\left(\mathfrak{d}\left(q^{*}, q_{n}\right)\right)\right]^{\varsigma_{1}}<\left[\vartheta\left(\mathfrak{d}\left(q^{*}, q_{n}\right)\right)\right]^{1-\varsigma_{2}} .
\end{aligned}
$$

This implies that

$$
\left[\vartheta\left(\mathfrak{D}\left(q_{n+1}, z\right)\right)\right]<\left[\vartheta\left(\mathfrak{D}\left(q^{*}, q_{n}\right)\right)\right]
$$

As $\vartheta$ is increasing, we have

$$
\mathfrak{d}\left(q_{n+1}, z\right)<\mathfrak{d}\left(q^{*}, q_{n}\right) .
$$

Then, by rectangular property, (51) and (72), we have

$$
\begin{aligned}
\mathfrak{d}(q, z) & \leq \mathfrak{d}\left(q^{*}, q_{n}\right)+\mathfrak{d}\left(q_{n}, q_{n+1}\right)+\mathfrak{d}\left(q_{n+1}, z\right) \\
& \leq \mathfrak{d}\left(q^{*}, q_{n}\right)+\frac{1}{n^{1 / Y}}+\mathfrak{d}\left(q^{*}, q_{n}\right) .
\end{aligned}
$$

Taking limit $n \longrightarrow \infty$ in the above inequality, we conclude that $z=q^{*}$. Hence,

$$
\mathfrak{d}\left(\mathfrak{g} q^{*}, \mathfrak{J} q^{*}\right)=\mathfrak{d}(\mathcal{Q}, \mathscr{P}) .
$$

Thus, $q^{*}$ is a coincidence best proximity point of pair of mappings $(\mathfrak{F}, \mathfrak{g})$.

Uniqueness. Now we have to show that $q^{*}$ is a unique coincidence best proximity point of pair of mappings $(\mathfrak{F}, \mathfrak{g})$. Suppose that $q^{*}$ and $w^{*}$ be two coincidence best proximity points of a pair of mappings $(\mathfrak{F}, \mathfrak{g})$, that is,

$$
\mathfrak{d}\left(\mathfrak{g} q^{*}, \mathfrak{F} q^{*}\right)=\mathfrak{d}(\mathcal{Q}, \mathscr{P})=\mathfrak{d}\left(\mathfrak{g} w^{*}, \mathfrak{J} w^{*}\right)
$$

Since $\alpha(q, p) \geq 0$, for every $q, p \in \mathbb{Q}$, and by using proper- ties of $\mathfrak{F}$ and $\mathfrak{g}$ and reasoning as above, we obtain that

$$
\begin{aligned}
{\left[\mathfrak{\vartheta}\left(\mathfrak{d}\left(\mathfrak{g} q^{*}, \mathfrak{g} w^{*}\right)\right)\right] \leq } & \mathfrak{\vartheta}\left(\mathfrak{d}\left(\mathfrak{J} q^{*}, \mathfrak{F} w^{*}\right)\right) \leq \alpha\left(q^{*}, w^{*}\right) \\
& +\vartheta\left(\mathfrak{d}\left(\mathfrak{J} q^{*}, \mathfrak{F} w^{*}\right)\right) \leq\left[\vartheta\left(\mathfrak{d}\left(q^{*}, w^{*}\right)\right)\right]^{\varsigma_{1}} \\
& \cdot\left[\vartheta\left(\mathfrak{d}\left(\mathfrak{g} q^{*}, \mathfrak{g} w^{*}\right)\right)\right]^{\varsigma_{2}}\left[\vartheta\left(\mathfrak{d}\left(\mathfrak{g} q^{*}, \mathfrak{g} w^{*}\right)\right)\right]^{1-\varsigma_{2}} \\
\leq & {\left[\vartheta\left(\mathfrak{D}\left(q^{*}, w^{*}\right)\right)\right]^{\varsigma_{1}}<\left[\vartheta\left(\mathfrak{D}\left(q^{*}, w^{*}\right)\right)\right]^{1-\varsigma_{2}} . }
\end{aligned}
$$

Further, we have

$\left[\vartheta\left(\mathfrak{d}\left(q^{*}, w^{*}\right)\right)\right]^{1-\varsigma_{2}} \leq\left[\vartheta\left(\mathfrak{D}\left(\mathfrak{g} q^{*}, \mathfrak{g} w^{*}\right)\right)\right]^{1-\varsigma_{2}}<\left[\vartheta\left(\mathfrak{D}\left(q^{*}, w^{*}\right)\right)\right]^{1-\varsigma_{2}}$,

which is a contradiction. Therefore, $q^{*}=w^{*}$. Hence, pair of mappings $(\mathfrak{F}, \mathfrak{g})$ has a unique coincidence best proximity point.

If $\mathfrak{g}$ is an isometry in Theorem 21 then it yields the following theorem.

Theorem 22. Let $\alpha: \mathbb{Q} \times \mathbb{Q} \longrightarrow-\infty, \infty),(\mathfrak{F}, \mathfrak{g})$ be a pair of mappings satisfying $\left(\mathcal{\vartheta}, \alpha^{+}, \mathfrak{g}\right)$-proximal contraction, where $\mathfrak{F}: \mathbb{Q} \longrightarrow \mathscr{P}$ be a triangular proximal $\alpha^{+}$-admissible and $\mathfrak{g}: \mathbb{Q} \longrightarrow \mathbb{Q}$ be a one to one isometry mapping satisfying $\alpha_{R}$ property. Moreover, if $Q_{0}$ is a nonempty $\alpha$-regular closed set in $\mathscr{X}, \mathfrak{F}\left(\mathcal{Q}_{0}\right) \subseteq \mathscr{P}_{0}, \mathscr{Q}_{0} \subseteq \mathfrak{g}\left(\mathscr{Q}_{0}\right)$, and $(\mathscr{Q}, \mathscr{P})$ satisfy the weak P-property. Then, there exists a coincidence best proximity point of the pair of mappings ( $\mathfrak{F}, \mathfrak{g})$ provided that there exists $q_{0}, q_{1} \in \mathbb{Q}_{0}$ such that

$$
\mathfrak{d}\left(\mathfrak{g} q_{1}, \mathfrak{F} q_{0}\right)=\mathfrak{d}(\mathscr{Q}, \mathscr{P}) \text { and } \alpha\left(q_{0}, q_{1}\right) \geq 0
$$

Moreover, if $\alpha(q, p) \geq 0$, for every $q, p \in \mathbb{Q}$ such that $\mathfrak{d}$ $\mathfrak{g} q, \mathfrak{J} q)=\mathfrak{d}(\mathbb{Q}, \mathscr{P})=\mathfrak{d}(\mathfrak{g} p, \mathfrak{F} p)$ then $q^{*}$ is the unique coincidence best proximity point of the pair of mappings $(\mathfrak{F}, \mathfrak{g})$.

Proof. The result follows from Theorem 21 by choosing $\mathfrak{g}$ as an isometry mapping instead of expansive mapping, and the remaining proof follows under the same lines.

Corollary 23. Let $\mathfrak{F}: \mathbb{Q} \longrightarrow \mathscr{P}$ be a $(\vartheta, \mathfrak{g})$-proximal contraction and $\mathfrak{g}: \mathbb{Q} \longrightarrow \mathbb{Q}$ be a one to one expansive mapping satisfying $q_{R}$ property. Moreover, if $Q_{0}$ is a nonempty closed set in $\mathscr{X}, \mathfrak{F}\left(\mathscr{Q}_{0}\right) \subseteq \mathscr{P}_{0}, \mathbb{Q}_{0} \subseteq \mathfrak{g}\left(\mathscr{Q}_{0}\right)$, and $(\mathbb{Q}, \mathscr{P})$ satisfy the weak $P$ -property. Then, there exists a unique coincidence best proximity point of pair of mappings $(\mathfrak{F}, \mathfrak{g})$ provided that there exists $q_{0}, q_{1} \in Q_{0}$ such that

$$
\mathfrak{d}\left(\mathfrak{g} q_{1}, \mathfrak{F} q_{0}\right)=\mathfrak{d}(\mathscr{Q}, \mathscr{P})
$$

Proof. The result follows from Theorem 21 by choosing $\alpha(q, p)=0$, and the remaining proof follows under the same lines.

Corollary 24. Let $\alpha: \mathbb{Q} \times \mathbb{Q} \longrightarrow-\infty, \infty)$ and $\mathfrak{F}: \mathbb{Q} \longrightarrow \mathscr{P}$ be a triangular proximal $\alpha^{+}$-admissible and $\left(\vartheta, \alpha^{+}\right)$-proximal contraction. Moreover, if $\mathbb{Q}_{0}$ is a nonempty $\alpha$-regular closed 
set, $\mathfrak{F}\left(\mathscr{Q}_{0}\right) \subseteq \mathscr{P}_{0}$ and $(\mathscr{Q}, \mathscr{P})$ satisfy the weak P-property. Then, there exists a best proximity point of $\mathfrak{F}$ provided that there exists $q_{0}, q_{1} \in \mathbb{Q}_{0}$ such that

$$
\mathfrak{d}\left(q_{1}, \mathfrak{\mho} q_{0}\right)=\mathfrak{d}(\mathscr{Q}, \mathscr{P}) \text { and } \alpha\left(q_{0}, q_{1}\right) \geq 0
$$

Moreover, if $\alpha(q, p) \geq 0$, for every $q, p \in \mathbb{Q}$ such that $\mathfrak{d}(q$, $\mathfrak{\Im} q)=\mathfrak{d}(\mathscr{Q}, \mathscr{P})=\mathfrak{d}(p, \mathfrak{\Im} p)$ then $q^{*}$ is a unique best proximity point of $\mathfrak{F}$.

Proof. The result follows from Theorem 21 by choosing $\mathfrak{g}=$ $I_{Q}$, and the remaining proof follows under the same lines.

Corollary 25. Let $\mathfrak{F}: \mathbb{Q} \longrightarrow \mathscr{P}$ be a 9 -proximal contraction. Moreover, if $\mathbb{Q}_{0}$ is a nonempty closed set, $\mathfrak{F}\left(\mathscr{Q}_{0}\right) \subseteq \mathscr{P}_{0}$ and $(\mathbb{Q}, \mathscr{P})$ satisfy the weak P-property. Then, there exists a unique best proximity point of $\mathfrak{F}$ provided that there exists $q_{0}, q_{1} \in \mathbb{Q}_{0}$ such that

$$
\mathfrak{d}\left(q_{1}, \mathfrak{\mho} q_{0}\right)=\mathfrak{d}(\mathscr{Q}, \mathscr{P})
$$

Proof. The result follows from Theorem 21 by choosing $\mathfrak{g}$ $=I_{Q}$ and $\alpha(q, p)=0$, and the remaining proof follows under the same lines.

To support the Corollary 25, we provide the following example.

Example 2. Let $\mathscr{X}=\{0,1,2,3,4,5,6,7,8\}$ and $\mathfrak{d}: \mathscr{X} \times \mathscr{X}$ $\longrightarrow 0, \infty)$ which is defined as

$$
\begin{gathered}
\mathfrak{D}(q, p)=\mathfrak{d}(p, q) \text { and } \mathfrak{d}(q, q)=0, \text { for all } q, p \in \mathscr{X}, \\
\mathfrak{d}(5,7)=1, \\
\mathfrak{d}(2,6)=\mathfrak{d}(1,3)=2, \mathfrak{d}(0,2)=3, \\
\mathfrak{d}(3,7)=\mathfrak{d}(2,4)=\mathfrak{d}(3,5)=4, \mathfrak{d}(0,4)=\mathfrak{d}(1,5)=5, \\
\mathfrak{d}(2,7)=\mathfrak{d}(4,7)=\mathfrak{d}(6,1)=\mathfrak{d}(6,5)=\mathfrak{d}(6,7)=\mathfrak{d}(2,5)=7, \\
\mathfrak{d}(4,6)=\mathfrak{d}(0,8)=\mathfrak{d}(2,8)=\mathfrak{d}(4,8)=\mathfrak{d}(6,8)=\mathfrak{d}(1,8)=\mathfrak{d}(4,1)=8, \\
\mathfrak{d}(0,1)=\mathfrak{d}(2,3)=\mathfrak{d}(4,5)=\mathfrak{d}(6,7)=\mathfrak{d}(0,5)=\mathfrak{d}(0,7)=\mathfrak{d}(2,1)=7, \\
\mathfrak{d}(0,6)=\mathfrak{d}(1,7)=\mathfrak{d}(6,3)=\mathfrak{d}(4,3)=\mathfrak{d}(0,3)=\mathfrak{d}(3,8)=\mathfrak{d}(5,8)=\mathfrak{d}(7,8)=8 .
\end{gathered}
$$

Then, $(\mathscr{X}, \mathfrak{d})$ is a Branciari metric space. Since

$$
\mathfrak{d}(0,6) \leq \mathfrak{d}(0,2)+\mathfrak{d}(2,6)
$$

then $(\mathscr{X}, \mathfrak{D})$ is not a metric space.

Suppose that $\mathbb{Q}=\{2,4,6\}$ and $\mathscr{P}=\{1,3,7\}$ are subsets of Branciari metric space $(\mathscr{X}, \mathfrak{d})$. Note that $\mathfrak{d}(\mathscr{Q}, \mathscr{P})=7$, $\mathbb{Q}=\mathbb{Q}_{0}$, and $\mathscr{P}=\mathscr{P}_{0}$. It is easy to see that $(\mathscr{Q}, \mathscr{P})$ satisfy the weak $P$-property. Define a mapping $\mathfrak{F}: \mathscr{Q} \longrightarrow \mathscr{P}$ as follows:

$$
\mathfrak{F}(2)=3, \mathfrak{F}(4)=1, \mathfrak{F}(6)=3
$$

Clearly, $\mathfrak{F}$ has no fixed point; also, note that $\mathfrak{F}\left(\mathscr{Q}_{0}\right) \subseteq \mathscr{P}_{0}$.
Define a function $\vartheta:(0, \infty) \longrightarrow(1, \infty)$ as follows:

$$
\vartheta(\kappa)=e^{\sqrt{\kappa}} .
$$

Hence, $\mathfrak{F}$ satisfies the conditions of 9 -proximal contractive mapping. Further, by taking $u=2, v=6, q=6, p=4 \in \mathbb{Q}$, $\varsigma_{1}=1 / 2$, and $\varsigma_{2} \geq 0$ in such a way that $0 \leq \varsigma_{1}+\varsigma_{2}<1$, we have

$$
\mathfrak{d}(2, \mathfrak{F}(6))=\mathfrak{d}(6, \mathfrak{F}(4))=7=\mathfrak{d}(\mathscr{Q}, \mathscr{P}) .
$$

This implies that $\mathfrak{F}$ satisfies

$$
\vartheta[\mathfrak{D}(\mathfrak{\mho}(q), \mathfrak{F}(p))] \leq[\mathfrak{\vartheta}(\mathfrak{d}(q, p))]^{1 / 2}[\vartheta(\mathfrak{d}(u, v))]^{\varsigma_{2}} .
$$

Hence, $\mathfrak{F}$ is $\mathfrak{\vartheta}$-proximal contraction for all $u, v, q, p \in \mathbb{Q}$. Thus, all conditions of Corollary 25 hold true; after simple calculation, we can find $q=2$ is a unique best proximity point of $\mathfrak{F}$.

Remark 26. If we take $\mathscr{X}=\mathscr{Q}=\mathscr{P}$ and $\varsigma_{2}=0$ in the Example 2 , we obtained the example of the main Theorem (1.7) in [4].

Theorem 27. Let $\alpha: \mathbb{Q} \times \mathbb{Q} \longrightarrow-\infty, \infty)$, (, $\mathfrak{g})$ be a pair of mappings satisfying $\left(\vartheta, \alpha^{+}, \mathfrak{g}\right)$-generalized proximal contraction, where $\mathfrak{F}: \mathbb{Q} \longrightarrow \mathscr{P}$ be a triangular proximal $\alpha^{+}$-admissible and $\mathfrak{g}: \mathbb{Q} \longrightarrow \mathbb{Q}$ be a one to one expansive mapping satisfying $\alpha_{R}$ property. Moreover, if $\mathbb{Q}_{0}$ is a nonempty $\alpha$-regular closed set in $\mathscr{X}, \mathfrak{F}\left(\mathscr{Q}_{0}\right) \subseteq \mathscr{P}_{0}, \mathscr{Q}_{0} \subseteq \mathfrak{g}\left(\mathscr{Q}_{0}\right)$, and $(\mathscr{Q}, \mathscr{P})$ satisfy the weak P-property. Then, there exists a coincidence best proximity point of the pair of mappings $(\mathfrak{F}, \mathfrak{g})$ provided that there exists $q_{0}, q_{1} \in Q_{0}$ such that

$$
\mathfrak{D}\left(\mathfrak{g} q_{1}, \mathfrak{\mho} q_{0}\right)=\mathfrak{d}(\mathscr{Q}, \mathscr{P}) \text { and } \alpha\left(q_{0}, q_{1}\right) \geq 0 \text {. }
$$

Moreover, if $\alpha(q, p) \geq 0$, for every $q, p \in \mathbb{Q}$ such that $\mathbb{D}$ $(\mathfrak{g} q, \mathfrak{F} q)=\mathfrak{d}(\mathscr{Q}, \mathscr{P})=\mathfrak{d}(\mathfrak{g} p, \mathfrak{F} p)$ then $q^{*}$ is the unique coincidence best proximity point of the pair of mappings $(\mathfrak{F}, \mathfrak{g})$.

Proof. Following the arguments similar to those given in the proof of Theorem 21, we obtain a sequence $\left\{q_{n}\right\}$ in $Q_{0}$ such that

$$
\begin{gathered}
\mathfrak{d}\left(\mathfrak{g} q_{n}, \mathfrak{F} q_{n-1}\right)=\mathfrak{d}(\mathscr{Q}, \mathscr{P}), \\
\mathfrak{d}\left(\mathfrak{g} q_{n+1}, \mathfrak{F} q_{n}\right)=\mathfrak{d}(\mathscr{Q}, \mathscr{P}), \alpha\left(q_{n+1}, q_{n}\right) \geq 0, \text { for all } n \in \mathbb{N} \cup\{0\} .
\end{gathered}
$$

If $q_{n} \neq q_{n+1}$, for all $n \in \mathbb{N} \cup\{0\}$, also the pair of mappings $(\mathfrak{F}, \mathfrak{g})$ is $\left(\vartheta, \alpha^{+}, \mathfrak{g}\right)$-generalized proximal contraction and $\mathfrak{g}$ is an expansive mapping, we have

$$
\begin{aligned}
\vartheta\left(\mathfrak{d}\left(q_{n}, q_{n+1}\right)\right) & \leq \vartheta\left(\mathfrak{d}\left(\mathfrak{g} q_{n}, \mathfrak{g} q_{n+1}\right)\right) \leq \alpha\left(q_{n}, q_{n-1}\right)+\vartheta\left(\mathfrak{d}\left(\mathfrak{g} q_{n}, \mathfrak{g} q_{n+1}\right)\right) \\
& \leq\left[\vartheta\left(\mathfrak{d}\left(q_{n-1}, q_{n}\right)\right)\right]^{\varsigma_{1}}\left[\vartheta\left(\mathfrak{d}\left(q_{n+1}, q_{n}\right)\right)\right]^{\varsigma_{2}} .
\end{aligned}
$$

Further, the above inequality becomes

$$
\left[\vartheta\left(\mathfrak{d}\left(q_{n}, q_{n+1}\right)\right)\right]^{1-\varsigma_{2}} \leq\left[\vartheta\left(\mathfrak{d}\left(q_{n}, q_{n-1}\right)\right)\right]^{\varsigma_{1}} .
$$


Again by using the arguments similar to those given in the proof of Theorem 21, we obtain

$$
\mathfrak{d}\left(q_{n}, q_{n+1}\right) \leq \frac{1}{n^{1 / Y}}, \text { for all } n \geq n_{0} .
$$

Now suppose that $q_{n}=q_{m}$ for every $n, m \in \mathbb{N}$ such that $n=m$. If $q_{n} \neq q_{n+2}$, for all $n \in \mathbb{N} \cup\{0\}$, since the pair of mappings $(\mathfrak{F}, \mathfrak{g})$ is $\left(\vartheta, \alpha^{+}, \mathfrak{g}\right)$-generalized proximal contraction and $\mathfrak{g}$ is an expansive mapping, we have

$$
\begin{aligned}
{\left[\mathcal{\vartheta}\left(\mathfrak{d}\left(q_{n}, q_{n+2}\right)\right)\right] } & \leq \mathfrak{\vartheta}\left(\mathfrak{d}\left(\mathfrak{g} q_{n}, \mathfrak{g} q_{n+2}\right)\right) \\
& \leq\left[\vartheta\left(\mathfrak{d}\left(q_{n-1}, q_{n+1}\right)\right)\right]^{\varsigma_{1}}\left[\vartheta\left(\mathfrak{D}\left(q_{n+2}, q_{n}\right)\right)\right]^{\varsigma_{2}} .
\end{aligned}
$$

Further, the above inequality becomes

$$
\left[\vartheta\left(\mathfrak{D}\left(q_{n}, q_{n+2}\right)\right)\right]^{1-\varsigma_{2}} \leq\left[\vartheta\left(\mathfrak{d}\left(q_{n-1}, q_{n+1}\right)\right)\right]^{\varsigma_{1}}
$$

By using the arguments similar to those given in the proof of Theorem 21, we deduce that $\left\{q_{n}\right\}$ is a Cauchy sequence in $\mathcal{Q}_{0} \subseteq Q \subset \mathcal{Q}$ and

$$
\mathfrak{d}\left(\mathfrak{g} z, \mathfrak{\mho} q^{*}\right)=\mathfrak{d}(\mathscr{Q}, \mathscr{P})
$$

Since the pair of mappings $(\mathfrak{F}, \mathfrak{g})$ is $\left(\mathcal{\vartheta}, \alpha^{+}, \mathfrak{g}\right)$-generalized proximal contraction and $\mathfrak{g}$ is an expansive mapping, we have

$$
\begin{aligned}
\vartheta\left[\mathfrak{d}\left(q_{n}, z\right)\right] & \leq \vartheta\left[\mathfrak{d}\left(\mathfrak{g} q_{n}, \mathfrak{g} z\right)\right] \leq \alpha\left(q_{n}, q^{*}\right)+\vartheta\left[\mathfrak{d}\left(\mathfrak{g} q_{n}, \mathfrak{g} z\right)\right] \\
& \leq\left[\vartheta\left(\mathfrak{d}\left(q_{n-1}, q^{*}\right)\right)\right]^{\varsigma_{1}}\left[\vartheta\left(\mathfrak{d}\left(z, q_{n}\right)\right)\right]^{\varsigma_{2}} \\
\vartheta\left[\mathfrak{D}\left(q_{n}, z\right)\right]^{1-\varsigma_{2}} & \leq\left[\vartheta\left(\mathfrak{d}\left(q_{n-1}, q^{*}\right)\right)\right]^{\varsigma_{1}}<\left[\vartheta\left(\mathfrak{d}\left(q_{n-1}, q^{*}\right)\right)\right]^{1-\varsigma_{2}} .
\end{aligned}
$$

By using the arguments similar to those given in the proof of Theorem 21, we conclude that $q^{*}$ is a coincidence best proximity point of pair of mappings $(\mathfrak{F}, \mathfrak{g})$.

Uniqueness. Now we have to show that $q^{*}$ is a unique coincidence best proximity point of pair of mappings $(\mathfrak{F}, \mathfrak{g})$ . Suppose that $q^{*}$ and $w^{*}$ be two coincidence best proximity points of a pair of mappings $(\mathfrak{F}, \mathfrak{g})$, that is,

$$
\mathfrak{d}\left(\mathfrak{g} q^{*}, \mathfrak{F} q^{*}\right)=\mathfrak{d}(\mathscr{Q}, \mathscr{P})=\mathfrak{d}\left(\mathfrak{g} w^{*}, \mathfrak{F} w^{*}\right) .
$$

Since $\alpha(q, p) \geq 0$, for every $q, p \in Q$ and by using properties of $\mathfrak{F}$ and $\mathfrak{g}$ and reasoning as above, we obtain that

$$
\begin{aligned}
\mathfrak{\vartheta}\left(\mathfrak{D}\left(q^{*}, w^{*}\right)\right) & \leq \mathfrak{\vartheta}\left(\mathfrak{d}\left(\mathfrak{g} q^{*}, \mathfrak{g} w^{*}\right)\right) \leq \alpha\left(q^{*}, w^{*}\right)+\vartheta\left(\mathfrak{d}\left(\mathfrak{g} q^{*}, \mathfrak{g} w^{*}\right)\right) \\
& \leq\left[\vartheta\left(\mathfrak{D}\left(q^{*}, w^{*}\right)\right)\right]^{\varsigma_{1}}\left[\vartheta\left(\mathfrak{D}\left(q^{*}, w^{*}\right)\right)\right]^{\varsigma_{2}} \\
& =\left[\vartheta\left(\mathfrak{D}\left(q^{*}, w^{*}\right)\right)\right]^{\varsigma_{1}+\varsigma_{2}}<\vartheta\left(\mathfrak{d}\left(q^{*}, w^{*}\right)\right),
\end{aligned}
$$

which is a contradiction. Therefore, $q^{*}=w^{*}$. Hence, pair of mappings $(\mathfrak{F}, \mathfrak{g})$ has a unique coincidence best proximity point.
If $\mathfrak{g}$ is an isometry then the preceding theorem yields the following Theorem.

Theorem 28. Let $\alpha: \mathbb{Q} \times \mathbb{Q} \longrightarrow-\infty, \infty),(\mathfrak{F}, \mathfrak{g})$ be a pair of mappings satisfying $\left(\vartheta, \alpha^{+}, \mathfrak{g}\right)$-generalized proximal contraction, where $\mathfrak{F}: \mathbb{Q} \longrightarrow \mathscr{P}$ be a triangular proximal $\alpha^{+}$-admissible and $\mathfrak{g}: \mathbb{Q} \longrightarrow \mathbb{Q}$ be a one to one isometry mapping satisfying $\alpha_{R}$ property. Moreover, if a $Q_{0}$ is nonempty $\alpha$-regular closed set in $\mathscr{X}, \mathfrak{F}\left(\mathscr{Q}_{0}\right) \subseteq \mathscr{P}_{0}, \mathscr{Q}_{0} \subseteq \mathfrak{g}\left(\mathscr{Q}_{0}\right)$, and $(\mathscr{Q}, \mathscr{P})$ satisfy the weak P-property. Then, there exists a coincidence best proximity point of the pair of mappings ( $\mathfrak{F}, \mathfrak{g})$ provided that there exists $q_{0}, q_{1} \in Q_{0}$ such that

$$
\mathfrak{d}\left(\mathfrak{g} q_{1}, \mathfrak{F} q_{0}\right)=\mathfrak{d}(\mathscr{Q}, \mathscr{P}) \text { and } \alpha\left(q_{0}, q_{1}\right) \geq 0
$$

Moreover, if $\alpha(q, p) \geq 0$, for every $q, p \in \mathbb{Q}$ such that $\mathfrak{d}(\mathfrak{g}$ $q$, $\mathfrak{F} q)=\mathfrak{d}(\mathfrak{Q}, \mathscr{P})=\mathfrak{d}(\mathfrak{g} p, \mathfrak{J} p)$ then $q^{*}$ is the unique coincidence best proximity point of the pair of mappings $(\mathfrak{F}, \mathfrak{g})$.

Proof. The result follows from Theorem 27 by choosing $\mathfrak{g}$ as an isometry mapping instead of an expansive mapping, and the remaining proof follows under the same lines.

Corollary 29. Let $(\mathfrak{F}, \mathfrak{g})$ be a pair of mappings satisfying $(\vartheta$, $\left.\alpha^{+}, \mathfrak{g}\right)$-generalized proximal contraction and $\mathfrak{g}: \mathbb{Q} \longrightarrow \mathbb{Q}$ be an expansive mapping satisfying $\alpha_{R}$ property. Moreover, if $\mathbb{Q}_{0}$ is nonempty closed set in $\mathscr{X}, \mathfrak{F}\left(\mathbb{Q}_{0}\right) \subseteq \mathscr{P}_{0}, \mathbb{Q}_{0} \subseteq \mathfrak{g}\left(\mathscr{Q}_{0}\right)$, and $(Q, \mathscr{P})$ satisfy the weak P-property. Then, there exists a unique coincidence best proximity point of the pair of mappings $(\mathfrak{F}, \mathfrak{g})$ provided that there exists $q_{0}, q_{1} \in \mathcal{Q}_{0}$ such that

$$
\mathfrak{d}\left(\mathfrak{g} q_{1}, \mathfrak{F} q_{0}\right)=\mathfrak{d}(\mathscr{Q}, \mathscr{P})
$$

Proof. The result follows from Theorem 27 by choosing $\alpha(q$, $p)=0$, and the remaining proof follows under the same lines.

Corollary 30. Let $\alpha: \mathbb{Q} \times \mathbb{Q} \longrightarrow-\infty, \infty), \mathfrak{F}: \mathbb{Q} \longrightarrow \mathscr{P}$ be a $\left(\vartheta, \alpha^{+}\right)$-generalized proximal contraction. Moreover, if $\mathbb{Q}_{0}$ is a nonempty $\alpha$-regular closed set in $\mathscr{X}, \mathfrak{F}\left(\mathcal{Q}_{0}\right) \subseteq \mathscr{P}_{0}$ and $(\mathbb{Q}$, $\mathscr{P})$ satisfy the weak $P$-property. Then, there exists a best proximity point of mapping $\mathfrak{F}$ provided that there exists $q_{0}, q_{1} \in \mathbb{Q}_{0}$ such that

$$
\mathfrak{d}\left(q_{1}, \mathfrak{\mho} q_{0}\right)=\mathfrak{d}(\mathscr{Q}, \mathscr{P}) \text { and } \alpha\left(q_{0}, q_{1}\right) \geq 0
$$

Moreover, if $\alpha(q, p) \geq 0$, for every $q, p \in \mathbb{Q}$ such that $\mathfrak{d}(q$, $\mathfrak{F} q)=\mathfrak{d}(\mathscr{Q}, \mathscr{P})=\mathfrak{d}(p, \mathfrak{F} p)$ then $q^{*}$ is the unique best proximity point of the mapping $\mathfrak{F}$.

Proof. The result follows from Theorem 27 by choosing $\mathfrak{g}=$ $I_{\mathscr{Q}}$, and the remaining proof follows under the same lines.

Corollary 31. Let $\mathfrak{F}: \mathbb{Q} \longrightarrow \mathscr{P}$ be a 9 -generalized proximal contraction. Moreover, if $\mathbb{Q}_{0}$ is a nonempty closed set, $\mathfrak{F}\left(\mathbb{Q}_{0}\right)$ $\subseteq \mathscr{P}_{0}$ and $(\mathcal{Q}, \mathscr{P})$ satisfy the weak P-property. Then, there exists a unique best proximity point of $\mathfrak{F}$ provided that there 
exists $q_{0}, q_{1} \in Q_{0}$ such that

$$
\mathfrak{d}\left(q_{1}, \mathfrak{J} q_{0}\right)=\mathfrak{d}(\mathscr{Q}, \mathscr{P})
$$

Proof. The result follows from Theorem 27 by choosing $\mathfrak{g}=$ $I_{\mathscr{Q}}$ and $\alpha(q, p)=0$, and the remaining follows under the same lines.

To support the Corollary 31, we provide the following example.

Example 3. Let $\mathscr{X}=\{0,1,2,3,4,5,6,7,8\}$ and $\mathfrak{d}: \mathscr{X} \times \mathscr{X}$ $\longrightarrow 0, \infty)$ which is defined as

$$
\mathfrak{D}(q, p)=\mathfrak{d}(p, q) \text { and } \mathfrak{d}(q, q)=0 \text {, for all } q, p \in \mathscr{X} \text {, }
$$

where

$$
\begin{gathered}
\mathfrak{d}(5,7)=1, \\
\mathfrak{d}(2,6)=\mathfrak{d}(1,3)=2, \mathfrak{d}(0,2)=3, \\
\mathfrak{d}(3,7)=\mathfrak{d}(2,4)=\mathfrak{d}(3,5)=4, \mathfrak{d}(0,4)=\mathfrak{d}(1,5)=5, \\
\mathfrak{d}(2,7)=\mathfrak{d}(6,1)=\mathfrak{d}(6,5)=\mathfrak{d}(6,7)=\mathfrak{d}(2,5)=7, \\
\mathfrak{d}(4,6)=\mathfrak{d}(0,8)=\mathfrak{d}(4,7)=\mathfrak{d}(2,8)=\mathfrak{d}(4,8)=\mathfrak{d}(6,8)=\mathfrak{d}(1,8)=\mathfrak{d}(4,1)=8, \\
\mathfrak{d}(0,1)=\mathfrak{d}(2,3)=\mathfrak{d}(4,5)=\mathfrak{d}(6,7)=\mathfrak{d}(0,5)=\mathfrak{d}(0,7)=\mathfrak{d}(2,1)=7, \\
\mathfrak{d}(0,6)=\mathfrak{d}(1,7)=\mathfrak{d}(6,3)=\mathfrak{d}(4,3)=\mathfrak{d}(0,3)=\mathfrak{d}(3,8)=\mathfrak{d}(5,8)=\mathfrak{d}(7,8)=8 .
\end{gathered}
$$

Then, $(\mathscr{X}, \mathfrak{d})$ is a Branciari metric space. Since

$$
\mathfrak{d}(0,6) \leq \mathfrak{d}(0,2)+\mathfrak{d}(2,6),
$$

then $(\mathscr{X}, \mathfrak{d})$ is not a metric space. Suppose that $\mathbb{Q}=\{2,4,6\}$ and $\mathscr{P}=\{3,5,7\}$. Note that $\mathfrak{d}(\mathscr{Q}, \mathscr{P})=7, \mathscr{Q}=\mathscr{Q}_{0}$, and $\mathscr{P}=$ $\mathscr{P}_{0}$. Define a mapping $\mathfrak{F}: \mathbb{Q} \longrightarrow \mathscr{P}$ as

$$
\mathfrak{F}(2)=3, \mathfrak{F}(4)=7, \mathfrak{F}(6)=3 \text {. }
$$

Clearly, $\mathfrak{F}$ has no fixed point; also, note that $\mathfrak{F}\left(\mathbb{Q}_{0}\right) \subseteq \mathscr{P}_{0}$. Define a function $\vartheta:(0, \infty) \longrightarrow(1, \infty)$ as

$$
\vartheta(\kappa)=e^{\sqrt{\kappa}} .
$$

Hence, $\mathfrak{F}$ satisfies the conditions of $\vartheta$-generalized proximal contractive mapping. Further, by taking $u=2, v=6$, $q=6, p=4 \in \mathbb{Q}, \varsigma_{1}=1 / 2$, and $\varsigma_{2} \geq 0$ in such a way that $0 \leq \varsigma_{1}$ $+\varsigma_{2}<1$, we have

$$
\mathfrak{d}(u, \mathfrak{F} q)=\mathfrak{d}(2, \mathfrak{F}(6))=\mathfrak{d}(6, \mathfrak{F}(4))=\mathfrak{d}(v, \mathfrak{F} p)=7
$$

Further, $\mathfrak{F}$ satisfies

$$
\vartheta[\mathfrak{d}(u, v)] \leq[\vartheta(\mathfrak{d}(q, p))]^{\varsigma_{1}}[\vartheta(\mathfrak{d}(u, v))]^{\varsigma_{2}} .
$$

Hence, $\mathfrak{F}$ is 9 -generalized proximal contraction for all $u$ , $v, q, p \in \mathbb{Q}$. Thus, all conditions of Corollary 31 hold true; after simple calculation, we can find $q=2$ is a unique best proximity point of $\mathfrak{F}$.

\section{Application to Coincidence Point and Fixed Point Theory}

If we take $\mathbb{Q}=\mathscr{P}=\mathscr{X}$, then from Definition 13, triangular proximal $\alpha^{+}$-admissible implies

$$
\begin{aligned}
& \mathfrak{d}(u, \mathfrak{F} q)=\mathfrak{d}(\mathscr{Q}, \mathscr{P})=0 \text { implies } u=\mathfrak{F} q, \\
& \mathfrak{d}(v, \mathfrak{F} p)=\mathfrak{d}(\mathscr{Q}, \mathscr{P})=0 \text { implies } v=\mathfrak{F} p,
\end{aligned}
$$

which becomes

(P1)

$$
\alpha(q, p) \geq 0 \text { implies } \alpha(\mathfrak{F} q, \mathfrak{F} p) \geq 0
$$

$$
\left.\begin{array}{l}
\alpha(q, z) \geq 0 \\
\alpha(z, p) \geq 0
\end{array}\right\} \text { implies } \alpha(q, p) \geq 0 \text {, for all } q, p \in \mathscr{X} \text {. }
$$

Remark 32. Note that, for self mapping, every triangular proximal $\alpha^{+}$-admissible self mapping is triangular $\alpha^{+}$ -admissible mapping.

Remark 33. If $\mathfrak{F}, \mathfrak{g}: \mathscr{X} \longrightarrow \mathscr{X}$ then $(\mathcal{\vartheta}, \mathfrak{g})$-proximal contraction becomes

$$
[\vartheta(\mathfrak{D}(\mathfrak{F} q, \mathfrak{F} p))] \leq[\vartheta(\mathfrak{D}(q, p))]^{\varsigma_{1}}[\vartheta(\mathfrak{D}(\mathfrak{F} q, \mathfrak{F} p))]^{\varsigma_{2}},
$$

and $(\vartheta, \mathfrak{g})$-generalized proximal contraction becomes

$$
\vartheta(\mathfrak{d}(\mathfrak{F} q, \mathfrak{F} p)) \leq[\vartheta(\mathfrak{d}(q, p))]^{\varsigma_{1}}[\vartheta(\mathfrak{d}(v, u))]^{\varsigma_{2}},
$$

where $\vartheta \in \Delta_{\vartheta}, \varsigma \in(0,1), \mathfrak{g} u=\mathfrak{F} q$, and $\mathfrak{g} v=\mathfrak{F} p$, for all $q, p \in \mathscr{X}$.

Definition 34. A self mapping $\mathfrak{F}$ satisfying inequality (113) is called $(\vartheta, \mathfrak{F})$-contraction, and the mapping which satisfies inequality $(114)$ is called $(\vartheta, \mathfrak{F})$-generalized contraction.

Remark 35. If $\alpha: \mathscr{X} \times \mathscr{X} \longrightarrow[-\infty, \infty)$ and a self mapping $\mathfrak{F}$ on $\mathscr{X}$ is $\left(\vartheta, \alpha^{+}\right)$-proximal contraction and $\left(\vartheta, \alpha^{+}\right)$-generalized proximal contraction then $\alpha(q, p) \geq 0$ implies that

$$
\alpha(q, p)+\vartheta(\mathfrak{d}(\mathfrak{\mho} q, \mathfrak{F} p)) \leq[\vartheta(\mathfrak{d}(q, p))]^{\varsigma_{1}}[\vartheta(\mathfrak{d}(\mathfrak{F} q, \mathfrak{F} p))]^{\varsigma_{2}}
$$

where $\vartheta \in \Delta_{\vartheta}, \varsigma \in(0,1), u=\mathfrak{F} q$, and $v=\mathfrak{F} p$, for all $q, p \in \mathscr{X}$.

Definition 36. A self mapping $\mathfrak{F}$ on $\mathscr{X}$ and $\alpha: \mathscr{X} \times \mathscr{X}$ $\longrightarrow[-\infty, \infty)$ satisfying inequality $(115)$ is called $\left(\vartheta, \alpha^{+}, \mathfrak{F}\right)$ -contraction.

Corollary 37. Let $\mathscr{X}$ be a complete Branciari metric space. Let $\mathfrak{F}: \mathscr{X} \longrightarrow \mathscr{X}$ be a $(\vartheta, \mathfrak{g})$-proximal contraction. Then, the pair of mappings ( $\mathfrak{F}, \mathfrak{g})$ has a coincidence point. 
Proof. Let $\mathbb{Q}=\mathscr{P}=\mathscr{X}$. We show that $\mathfrak{F}$ satisfies $(\vartheta, \mathfrak{F})$-contraction. Then, we have

$$
\begin{aligned}
& \mathfrak{d}(\mathfrak{g} u, \mathfrak{\mho} q)=\mathfrak{d}(\mathscr{Q}, \mathscr{P})=0, \\
& \mathfrak{d}(\mathfrak{g} v, \mathfrak{F} p)=\mathfrak{d}(\mathscr{Q}, \mathscr{P})=0,
\end{aligned}
$$

for all $q, p, u, v \in \mathscr{X}$, since $\mathfrak{d}(\mathscr{Q}, \mathscr{P})=0$ implies that $\mathfrak{g} u=\mathfrak{F} q$ and $\mathfrak{g} v=\mathfrak{F} p$ and since $\mathfrak{F}$ satisfies condition (113). So,

$$
\begin{aligned}
\vartheta(\mathfrak{d}(\mathfrak{\mho} q, \mathfrak{F} p)) & \leq[\vartheta(\mathfrak{d}(q, p))]^{\varsigma_{1}}[\vartheta(\mathfrak{d}(\mathfrak{g} q, \mathfrak{g} p))]^{\varsigma_{2}} \\
& =[\vartheta(\mathfrak{d}(q, p))]^{\varsigma_{1}}[\vartheta(\mathfrak{d}(\mathfrak{J} q, \mathfrak{F} p))]^{\varsigma_{2}},
\end{aligned}
$$

which implies that

$$
\vartheta(\mathfrak{d}(\mathfrak{\Im} q, \mathfrak{\Im p} p)) \leq[\vartheta(\mathfrak{d}(q, p))]^{\varsigma_{1}}[\vartheta(\mathfrak{d}(\mathfrak{\mho} q, \mathfrak{F} p))]^{\varsigma_{2}},
$$

which further implies that $\mathfrak{F}$ is $(\vartheta, \mathfrak{F})$-contraction. As $\mathfrak{d}(\mathfrak{g} q$, $\mathfrak{F} q)=\mathfrak{d}(\mathscr{Q}, \mathscr{P})=0$ then $q$ is a coincidence point of $\mathfrak{F}$ and $\mathfrak{g}$.

Corollary 38. Let $\mathscr{X}$ be a complete Branciari metric space and pair of mapping $\mathfrak{F}, \mathfrak{g}: \mathscr{X} \longrightarrow \mathscr{X}$ be $a(\mathfrak{\vartheta}, \mathfrak{g})$-generalized proximal contraction. Then, pair of mappings $(\mathfrak{F}, \mathfrak{g})$ has a coincidence point.

Proof. Let $\mathbb{Q}=\mathscr{P}=\mathscr{X}$. We show that $\mathfrak{F}$ satisfies $(\vartheta, \mathfrak{F})$-generalized contraction. Then, we have

$$
\begin{aligned}
& \mathfrak{d}(\mathfrak{g} u, \mathfrak{\Im} q)=\mathfrak{d}(\mathscr{Q}, \mathscr{P})=0, \\
& \mathfrak{d}(\mathfrak{g} v, \mathfrak{F} p)=\mathfrak{d}(\mathfrak{Q}, \mathscr{P})=0,
\end{aligned}
$$

for all $q, p, u, v \in \mathscr{X}$, since $\mathfrak{d}(\mathscr{Q}, \mathscr{P})=0$ implies that $\mathfrak{g} u=\mathfrak{F} q$ and $\mathfrak{g} v=\mathfrak{F} p$ and since $\mathfrak{F}$ satisfies condition (114). So,

$$
\begin{aligned}
\vartheta(\mathfrak{d}(\mathfrak{J} q, \mathfrak{F} p)) & =\mathfrak{\vartheta}(\mathfrak{d}(\mathfrak{g} u, \mathfrak{g} v)) \leq[\vartheta(\mathfrak{d}(q, p))]^{\varsigma_{1}}[\vartheta(\mathfrak{d}(v, u))]^{\varsigma_{2}}, \\
& \leq[\vartheta(\mathfrak{d}(q, p))]^{\varsigma_{1}}[\vartheta(\mathfrak{d}(u, v))]^{\varsigma_{2}},
\end{aligned}
$$

which implies that

$$
\vartheta(\mathfrak{d}(\mathfrak{F} q, \mathfrak{F} p)) \leq[\vartheta(\mathfrak{d}(q, p))]^{\varsigma_{1}}[\vartheta(\mathfrak{d}(u, v))]^{\varsigma_{2}},
$$

which further implies that $\mathfrak{F}$ is $(\vartheta, \mathfrak{F})$-generalized contraction. As $\mathfrak{d}(\mathfrak{g} q, \mathfrak{F} q)=\mathfrak{d}(\mathscr{Q}, \mathscr{P})=0$ then $q$ is a coincidence point of $\mathfrak{F}$ and $\mathfrak{g}$.

If $\mathfrak{g}$ is an identity mapping then we will have the following corollary.

Corollary 39. Let $\mathscr{X}$ be a complete Branciari metric space, $\alpha: \mathscr{X} \times \mathscr{X} \longrightarrow[-\infty, \infty)$, and $\mathfrak{F}: \mathscr{X} \longrightarrow \mathscr{X}$ be a $\left(\vartheta, \alpha^{+}, \mathfrak{F}\right)$ -contraction. If $\left\{q_{n}\right\}$ is a sequence in $\mathscr{X}$ such that $\alpha\left(q_{n}\right.$, $\left.q_{n+1}\right) \geq 0$ and $q_{n} \longrightarrow q \in \mathscr{X}$ as $n \longrightarrow \infty$ then $\alpha\left(q_{n}, q\right) \geq 0$ for all $n \in \mathbb{N}$. Then, there exists a fixed point of $\mathfrak{F}$ provided that there exists $q_{0} \in \mathscr{X}$ such that $\alpha\left(q_{0}, \mathfrak{F} q_{0}\right) \geq 0$. Moreover, if $\alpha(q, p) \geq 0$, for all $q, p \in \mathfrak{F}(\mathscr{X})$ then $q^{*}$ is the unique fixed point of the mapping $\mathfrak{\mho}$.
Proof. Let $\mathbb{Q}=\mathscr{P}=\mathscr{X}$. We show that $\mathfrak{F}$ satisfies $\left(\vartheta, \alpha^{+}\right)$ -proximal contraction and $\left(\vartheta, \alpha^{+}\right)$-generalized proximal contraction. Then, we have

$$
\begin{aligned}
\alpha(q, p) & \geq 0, \\
\mathfrak{d}(u, \mathfrak{F} q) & =\mathfrak{d}(\mathscr{Q}, \mathscr{P})=0, \\
\mathfrak{d}(v, \mathfrak{F} p) & =\mathfrak{d}(\mathscr{Q}, \mathscr{P})=0,
\end{aligned}
$$

for all $q, p, u, v \in \mathscr{X}$, since $\mathfrak{d}(\mathscr{Q}, \mathscr{P})=0$ implies that $u=\mathfrak{F} q$ and $v=\mathfrak{F} p$ and since $\mathfrak{F}$ satisfies condition (115). So,

$$
\begin{aligned}
\alpha(q, p)+\vartheta(\mathfrak{d}(\mathfrak{F} q, \mathfrak{F} p)) & =\alpha(q, p)+\vartheta(\mathfrak{d}(\mathfrak{J} q, \mathfrak{F} p)) \\
& \leq[\vartheta(\mathfrak{d}(q, p))]^{\varsigma_{1}}[\vartheta(\mathfrak{d}(\mathfrak{J} q, \mathfrak{F} p))]^{\varsigma_{2}} \\
& =[\vartheta(\mathfrak{d}(q, p))]^{\varsigma_{1}}[\vartheta(\mathfrak{d}(u, v))]^{\varsigma_{2}},
\end{aligned}
$$

which implies that

$$
\alpha(q, p)+\vartheta(\mathfrak{d}(\mathfrak{F} q, \mathfrak{F} p)) \leq[\vartheta(\mathfrak{d}(\mathrm{q}, p))]^{\varsigma_{1}}[\vartheta(\mathfrak{d}(u, v))]^{\varsigma_{2}}
$$

which further implies that $\mathfrak{F}$ is $\left(\vartheta, \alpha^{+}\right)$-proximal contraction. Also, we have

$$
\begin{aligned}
& \alpha(q, p)+\vartheta(\mathfrak{d}(u, v)) \leq \alpha(q, p)+\vartheta(\mathfrak{d}(\mathfrak{J} q, \mathfrak{F} p)) \\
& \leq[\vartheta(\mathfrak{D}(q, p))]^{\varsigma_{1}}[\vartheta(\mathfrak{D}(\mathfrak{\mho} q, \mathfrak{F} p))]^{\varsigma_{2}} \\
& \leq[\vartheta(\mathfrak{D}(q, p))]^{\varsigma_{1}}[\vartheta(\mathfrak{D}(u, v))]^{\varsigma_{2}},
\end{aligned}
$$

which implies that

$$
\alpha(q, p)+\vartheta(\mathfrak{d}(u, v)) \leq[\vartheta(\mathfrak{d}(q, p))]^{\varsigma_{1}}[\vartheta(\mathfrak{d}(u, v))]^{\varsigma_{2}},
$$

which further implies that $\mathfrak{F}$ is $\left(\vartheta, \alpha^{+}\right)$-generalized proximal contraction. Since $\mathfrak{F}$ is triangular proximal $\alpha^{+}$-admissible, by Remark 32, $\mathfrak{F}$ is a triangular $\alpha^{+}$-admissible mapping. If there exists $q \in \mathscr{X}$ such that $\alpha(q, \mathfrak{F} q) \geq 0$ and $\mathfrak{d}(q, \mathfrak{F} q)=\mathfrak{d}(\mathscr{Q}$, $\mathscr{P})=0$, it gives that $q$ is a fixed point of $\mathfrak{F}$.

Uniqueness. Let $\alpha(q, p) \geq 0$ for all $q, p \in \mathfrak{F}(\mathscr{X})$. Now we will show that $q^{*}$ is a unique fixed point of $\mathfrak{F}$. On contrary, suppose that $w^{*}$ be another fixed point of mapping $\mathfrak{F}$ with $q^{*}=w^{*}$. Hence,

$$
\mathfrak{d}\left(\mathfrak{g} w^{*}, \mathfrak{F} w^{*}\right)=\mathfrak{d}(\mathscr{Q}, \mathscr{P})
$$

Then, by using the properties of $\mathfrak{F}$, we obtain that

$$
\begin{aligned}
\mathfrak{\vartheta}\left(\mathfrak{d}\left(q^{*}, w^{*}\right)\right) & =\mathfrak{\vartheta}\left(\mathfrak{d}\left(\mathfrak{F} q^{*}, \mathfrak{F} w^{*}\right)\right) \leq \alpha\left(q^{*}, w^{*}\right)+\vartheta\left(\mathfrak{d}\left(\mathfrak{J} q^{*}, \mathfrak{F} w^{*}\right)\right) \\
& \leq\left[\vartheta\left(\mathfrak{d}\left(q^{*}, w^{*}\right)\right)\right]^{\varsigma_{1}}\left[\vartheta\left(\mathfrak{d}\left(\mathfrak{J} q^{*}, \mathfrak{J} w^{*}\right)\right)\right]^{\varsigma_{2}} \\
& =\left[\vartheta\left(\mathfrak{d}\left(q^{*}, w^{*}\right)\right)\right]^{\varsigma_{1}}\left[\vartheta\left(\mathfrak{d}\left(q^{*}, w^{*}\right)\right)\right]^{\varsigma_{2}} \\
& =\left[\vartheta\left(\mathfrak{d}\left(q^{*}, w^{*}\right)\right)\right]^{\varsigma_{1}+\varsigma_{2}}<\left[\vartheta\left(\mathfrak{d}\left(q^{*}, w^{*}\right)\right)\right],
\end{aligned}
$$

which is a contradiction. Therefore, $q^{*}=w^{*}$. Hence, the mapping $\mathfrak{F}$ has a unique fixed point. 
Remark 40. By taking $\alpha(q, p)=0$ and $\varsigma_{2}=0$, for all $q, p \in \mathscr{X}$ in the above Corollary 39, we obtain the main Theorem (1.7) in [4] as Corollary.

\section{Application to Graph Theory}

Let $\mathscr{X}$ be a nonempty set and define a set $\Delta=\{(q, p) \in \mathscr{X} \times$ $\mathscr{X}, q, p \in \mathscr{X}\}$. A graph $\mathfrak{g}$ is a pair $(V, E)$, where $V=V(\mathfrak{g})$ is a set of vertices coinciding with $\mathscr{X}$ and $E=E(\mathfrak{g})$ is a set of its edges such that $\Delta \subset E(\mathfrak{g})$. Moreover, we suppose that the graph $\mathfrak{g}$ is without parallel edges. By reversing the direction of edges in $\mathfrak{g}$, we obtain a graph $\mathfrak{g}^{-1}$ whose edge set and vertex set are defined as follows:

$$
E\left(\mathfrak{g}^{-1}\right)=\left\{(q, p) \in \mathscr{X}^{2}:(p, q) \in E(\mathfrak{g})\right\} \text { and } V\left(\mathfrak{g}^{-1}\right)=V(\mathfrak{g})
$$

Consider the graph $\tilde{\mathfrak{g}}$ comprising of all vertices and edges of $\mathfrak{g}$ and $\mathfrak{g}^{-1}$, that is,

$$
E(\tilde{\mathfrak{g}})=E(\mathfrak{g}) \cup E\left(\mathfrak{g}^{-1}\right)
$$

We denote the undirected graph by $\tilde{\mathfrak{g}}$ obtained by ignoring the direction of edges of $\mathfrak{g}$.

Definition 41 (see [24]).

(1) A subgraph $H$ of a graph $\mathfrak{g}$ is consisting upon a subset of edges of graph $\mathfrak{g}$ and associated vertices.

(2) Let $q$ and $p$ be two vertices in a graph $\mathfrak{g}$. A path of length $n$ (where $n \in \mathbb{N} \cup\{0\}$ ) in $\mathfrak{g}$ from $q$ to $p$ is a sequence $\left(q_{i}\right)_{i=0}^{n}$ of $n+1$ distinct vertices such that $q_{0}=q, q_{n}=p$, and $\left(q_{i}, q_{i+1}\right) \in E(\mathfrak{g})$ for $i=1,2, \cdots, n$.

(3) A graph $\mathfrak{g}$ is called a connected graph if there exists a path between any two vertices of graph $\mathfrak{g}$, and it is said to be a weakly connected graph if $\tilde{\mathfrak{g}}$ is connected.

(4) A path is called elementary if no vertices appear more than one time in it.

Throughout this section, we suppose that $(\mathscr{X}, \mathfrak{d})$ be a Branciari metric space and a graph $\mathfrak{g}$ may be transformed to a weighed graph by appointing to each edge the distance given by the Branciari metric between its vertices. In order to apply the rectangular inequality to the vertices of the graph $\mathfrak{g}$, we will consider the graph of length greater than 2 , which signifies that between two vertices, we will obtain a path between two vertices.

Definition 42 . Let $(\mathcal{Q}, \mathscr{P})$ be a pair of nonempty subsets of a Branciari metric space $\mathscr{X}$ and $\mathfrak{g}$ be a directed graph without parallel edges such that $V(\mathfrak{g})=\mathscr{X}$. A mapping $\mathfrak{F}: \mathscr{Q} \longrightarrow \mathscr{P}$ is said to be $(\vartheta, \mathfrak{g})$-proximal contraction if for all $q, p \in \mathbb{Q}$, $q \neq p$ with $(q, p) \in E(\mathfrak{g})$ such that

$$
\left.\begin{array}{c}
\mathfrak{d}(u, \mathfrak{\mho} q)=\mathfrak{d}(\mathscr{Q}, \mathscr{P}) \\
\mathfrak{d}(v, \mathfrak{F} p)=\mathfrak{d}(\mathscr{Q}, \mathscr{P})
\end{array}\right\} \begin{gathered}
\text { implies }[ \\
{[(\mathfrak{d}(\mathfrak{\mho} q, \mathfrak{\mho} p))] \leq[\vartheta(\mathfrak{d}(q, p))]^{\varsigma_{1}}[\vartheta(\mathfrak{d}(v, u))]^{\varsigma_{2}},} \\
(u, v) \in E(\mathfrak{g}),
\end{gathered}
$$

where, $p, u, v \in \mathbb{Q}, \vartheta \in \Delta_{\vartheta}$, and $\varsigma_{1}, \varsigma_{2} \geq 0$ with $0<\varsigma_{1}+\varsigma_{2}<1$.

Definition 43 . Let $(\mathcal{Q}, \mathscr{P})$ be a pair of nonempty subsets of a Branciari metric space $\mathscr{X}$ and $\mathfrak{g}$ be a directed graph without parallel edges such that $V(\mathfrak{g})=\mathscr{X}$. A mapping $\mathfrak{F}: \mathbb{Q} \longrightarrow \mathscr{P}$ is said to be $(\vartheta, \mathfrak{g})$-generalized proximal contraction if for all $q, p \in \mathbb{Q}, q \neq p$ with $(q, p) \in E(\mathfrak{g})$ such that

$$
\left.\begin{array}{c}
\mathfrak{d}(u, \mathfrak{F} q)=\mathfrak{d}(\mathscr{Q}, \mathscr{P}) \\
\mathfrak{d}(v, \mathfrak{F} p)=\mathfrak{d}(\mathscr{Q}, \mathscr{P})
\end{array}\right\} \begin{gathered}
\text { implies }[\vartheta(\mathfrak{d}(u, v))] \leq[\vartheta(\mathfrak{d}(q, p))]^{\varsigma_{1}}[\vartheta(\mathfrak{d}(v, u))]^{\varsigma_{2}}, \\
(u, v) \in E(\mathfrak{g}),
\end{gathered}
$$

where, $p, u, v \in \mathbb{Q}, \vartheta \in \Delta_{\vartheta}$, and $\varsigma_{1}, \varsigma_{2} \geq 0$ with $0<\varsigma_{1}+\varsigma_{2}<1$.

Corollary 44. Let $\mathfrak{F}: \mathbb{Q} \longrightarrow \mathscr{P}$ a $(\vartheta, \mathfrak{g})$-proximal contraction. If $\mathfrak{F}\left(\mathscr{Q}_{0}\right) \subseteq \mathscr{P}_{0}$ and $\mathbb{Q}_{0}$ is nonempty closed subset in $\mathscr{X}$, and $(\mathcal{Q}, \mathscr{P})$ satisfy the weak P-property. Also, there exists $q_{0}, q_{1} \in \mathbb{Q}_{0}$ such that there exists an elementary path between them and

$$
\mathfrak{d}\left(q_{1}, \mathfrak{F} q_{0}\right)=\mathfrak{d}(\mathscr{Q}, \mathscr{P})
$$

Moreover, if there exists a path $\left(\mathfrak{v}_{i}^{\varsigma}\right)_{i, \varsigma=0}^{N} \subseteq Q_{0}$ in $\mathfrak{g}$ between any two elements $q$ and $p$ then $\mathfrak{F}$ has a unique best proximity point.

Proof. Let $q_{0}, q_{1} \in \mathbb{Q}_{0}$ such that $\mathfrak{d}\left(q_{1}, \mathfrak{J} q_{0}\right)=\mathfrak{d}(\mathscr{Q}, \mathscr{P})$. A path $\left(\mathfrak{v}_{0}^{i}\right)_{i=0}^{N}$ in $\mathfrak{g}$ is a sequence containing points of $\widehat{Q}_{0}$. Consequently, $\mathfrak{v}_{0}^{0}=q_{0}, \mathfrak{v}_{0}^{N}=q_{1}$ and $\left(\mathfrak{v}_{0}^{i}, \mathfrak{v}_{0}^{i+1}\right) \in E(\mathfrak{g})$ for all 0 $\leq i \leq N$. Given that $\mathfrak{v}_{0}^{1} \in \mathbb{Q}_{0}$, since $\mathfrak{F}\left(\mathscr{Q}_{0}\right) \subseteq \mathscr{P}_{0}$ and from definition of $\mathbb{Q}_{0}$, there exists $\mathfrak{v}_{1}^{1} \in \mathcal{Q}_{0}$ such that $\mathfrak{d}\left(\mathfrak{v}_{1}^{1}, \mathfrak{F} \mathfrak{w}_{0}^{1}\right)=\mathfrak{d}(\mathbb{Q}$, $\mathscr{P})$. Similarly, for each $i=2, \cdots, N$, there exists $\mathfrak{b}_{1}^{i} \in \mathbb{Q}_{0}$ such that $\mathfrak{d}\left(\mathfrak{v}_{1}^{i}, \mathfrak{F}_{0}^{i}\right)=\mathfrak{d}(\mathscr{Q}, \mathscr{P})$. As $\left(\mathfrak{v}_{0}^{i}\right)_{i=0}^{N}$ is a path in $\mathfrak{g}$ then $\left(\mathfrak{v}_{0}^{0}\right.$, $\left.\mathfrak{v}_{0}^{1}\right)=\left(q_{0}, \mathfrak{b}_{0}^{1}\right) \in E(\mathfrak{g})$. From the above argument, we have $\mathfrak{d}$ $\left(q_{1}, \mathfrak{F} q_{0}\right)=\mathfrak{d}(\mathscr{Q}, \mathscr{P})$ and $\mathfrak{d}\left(\mathfrak{v}_{1}^{1}, \mathfrak{F} \mathfrak{v}_{0}^{1}\right)=\mathfrak{d}(\mathscr{Q}, \mathscr{P})$. Since, $\mathfrak{F}$ is $(\vartheta, \mathfrak{g})$-proximal contraction, it follows that $\left(q_{1}, \mathfrak{v}_{1}^{1}\right) \in E(\mathfrak{g})$. In similar manner, we have the following:

$$
\left(\mathfrak{v}_{1}^{i-1}, \mathfrak{v}_{1}^{i}\right) \in E(\mathfrak{g}) \text {, for all } i=1,2, \cdots, N
$$

Let $q_{2}=\mathfrak{v}_{1}^{N}$ then $\left(\mathfrak{v}_{1}^{i}\right)_{i=0}^{N}$ is a path from $q_{1}=\mathfrak{v}_{1}^{0}$ to $q_{2}=\mathfrak{v}_{1}^{N}$. For each $i=1,2,3, \cdots, N$, as $\mathfrak{v}_{1}^{i} \in \mathscr{Q}_{0}$ and $\mathfrak{F}_{1}^{i} \in \mathfrak{F}\left(\mathscr{Q}_{0}\right) \subseteq \mathscr{P}_{0}$ then by definition of $\mathscr{P}_{0}$, there exists $\mathfrak{v}_{2}^{i} \in \mathscr{Q}_{0}$ such that $\mathfrak{d}\left(\mathfrak{v}_{2}^{i}\right.$, $\left.\mathfrak{F} \mathfrak{v}_{1}^{i}\right)=\mathfrak{d}(\mathscr{Q}, \mathscr{P})$. In addition, we have $\mathfrak{d}\left(q_{2}, \mathfrak{F} q_{1}\right)=\mathfrak{d}(\mathscr{Q}, \mathscr{P})$. 
As above mentioned, we have

$$
\left(q_{2}, \mathfrak{v}_{2}^{1}\right) \in E(\mathfrak{g}) \text { and }\left(\mathfrak{v}_{2}^{i-1}, \mathfrak{v}_{2}^{i}\right) \in E(\mathfrak{g}) \text {, for all } i=1,2 \cdots, N
$$

Similarly, by $\mathfrak{F}\left(\mathbb{Q}_{0}\right) \subseteq \mathscr{P}_{0}$, there exists a point $q_{3} \in \mathbb{Q}_{0}$ where $q_{3}=\mathfrak{v}_{2}^{N}$. Then, $\left(\mathfrak{v}_{2}^{i}\right)_{i=0}^{N}$ is a path from $\mathfrak{v}_{2}^{0}=q_{2}$ and $\mathfrak{v}_{2}^{N}=$ $q_{3}$. Continuing this way, we can obtain a sequence $\left\{q_{n}\right\}_{n \in \mathbb{N}}$ where $q_{n+1} \in\left[q_{n}\right]_{\mathfrak{g}}^{N}$ and $\mathfrak{d}\left(q_{n+1}, \mathfrak{F} q_{n}\right)=\mathfrak{d}(\mathscr{Q}, \mathscr{P})$ by producing a path $\left(\mathfrak{v}_{n}^{i}\right)_{i=0}^{N}$ from $q_{n}=\mathfrak{v}_{\mathrm{n}}^{0}$ and $q_{n+1}=\mathfrak{v}_{n}^{N}$ in such a way that

$$
\mathfrak{d}\left(\mathfrak{v}_{n+1}^{i}, \mathfrak{F}_{n}^{i}\right)=\mathfrak{d}(\mathscr{Q}, \mathscr{P})
$$

for all $i=0,1, \cdots, N, n \in \mathbb{N}$. Thus, we have

$\mathfrak{d}\left(\mathfrak{v}_{n}^{i-1}, \mathfrak{F}_{\mathfrak{v}^{i-1}}^{i-1}\right)=\mathfrak{d}(\mathscr{Q}, \mathscr{P})=\mathfrak{d}\left(\mathfrak{v}_{n}^{i}, \mathfrak{F}_{\mathfrak{p}^{i}-1}^{i}\right)$, for all $i=1,2, \cdots, N, n \in \mathbb{N}$

Next, we claim that $\mathfrak{d}\left(q_{n}, q_{n+1}\right) \leq C / n^{1 / Y}$, where $C$ is a constant. To prove the claim, we need to consider the following two cases where $\left(\mathfrak{v}_{n}^{i}\right)_{i=0,1, \cdots, N}$ is a path from $q_{n}$ to $q_{n+1}$. Note that for all $i=0,1, \cdots, N\left(\mathbf{v}_{n}^{i}\right)_{i=0,1, \cdots, N}$ are different owing to the fact that the considered path $\left(\mathfrak{v}_{n}^{i}\right)$ is elementary. Then, we can apply the rectangular inequality.

Case $1 . N=2 k+1$ ( $N$ is odd). For any positive integer $n$, we get

$$
\begin{aligned}
\mathfrak{D}\left(q_{n}, q_{n+1}\right) & =\mathfrak{d}\left(\mathfrak{v}_{n}^{0}, \mathfrak{v}_{n}^{N}\right)=\mathfrak{d}\left(\mathfrak{v}_{n}^{0}, \mathfrak{v}_{n}^{2 k+1}\right) \\
& \leq \mathfrak{d}\left(\mathfrak{v}_{n}^{0}, \mathfrak{v}_{n}^{1}\right)+\mathfrak{d}\left(\mathfrak{v}_{n}^{1}, \mathfrak{v}_{n}^{2}\right)+\mathfrak{d}\left(\mathfrak{v}_{n}^{2}, \mathfrak{v}_{n}^{2 k+1}\right) \\
& \leq \mathfrak{d}\left(\mathfrak{v}_{n}^{0}, \mathfrak{v}_{n}^{1}\right)+\mathfrak{d}\left(\mathfrak{v}_{n}^{1}, \mathfrak{v}_{n}^{2}\right)+\cdots+\mathfrak{d}\left(\mathfrak{v}_{n}^{2 k}, \mathfrak{v}_{n}^{2 k+1}\right) \\
& \leq \sum_{i=1}^{2 k+1} \mathfrak{d}\left(\mathfrak{v}_{n}^{i-1}, \mathfrak{v}_{n}^{i}\right) .
\end{aligned}
$$

Since $\mathfrak{F}$ is $(\mathcal{\vartheta}, \mathfrak{g})$-proximal contraction, we have

$$
\sum_{i=1}^{2 k+1} \mathfrak{\vartheta}\left(\mathfrak{d}\left(\mathfrak{F}_{n-1}^{i-1}, \mathfrak{F}_{n-1}^{i}\right)\right) \leq \sum_{i=1}^{2 k+1}\left(\left[\mathcal{\vartheta}\left(\mathfrak{d}\left(\mathfrak{v}_{n-1}^{i-1}, \mathfrak{v}_{n-1}^{i}\right)\right)\right]^{\varsigma_{1}}\left[\mathcal{\vartheta}\left(\mathfrak{d}\left(\mathfrak{v}_{n-1}^{i-1}, \mathfrak{v}_{n-1}^{i}\right)\right)\right]^{\varsigma_{2}}\right) .
$$

Since pair of subsets $(\mathcal{Q}, \mathscr{P})$ satisfies the weak $P$-property then we have

$$
\sum_{i=1}^{2 k+1} \mathfrak{d}\left(\mathfrak{v}_{n}^{i-1}, \mathfrak{v}_{n}^{i}\right) \leq \sum_{i=1}^{2 k+1} \mathfrak{d}\left(\mathfrak{F v}_{n-1}^{i-1}, \mathfrak{F v}_{n-1}^{i}\right), \text { for all } n \in \mathbb{N}
$$

Then, from above inequalities, we have

$$
\begin{aligned}
\sum_{i=1}^{2 k+1} \mathfrak{\vartheta}\left(\mathfrak{d}\left(\mathfrak{v}_{n}^{i-1}, \mathfrak{v}_{n}^{i}\right)\right) \leq & \sum_{i=1}^{2 k+1}\left(\left[\vartheta\left(\mathfrak{d}\left(\mathfrak{v}_{n-1}^{i-1}, \mathfrak{v}_{n-1}^{i}\right)\right)\right]^{\varsigma_{1}}\left[\vartheta\left(\mathfrak{d}\left(\mathfrak{v}_{n-1}^{i-1}, \mathfrak{v}_{n-1}^{i}\right)\right)\right]^{\varsigma_{2}}\right) \\
& \cdot \sum_{i=1}^{2 k+1}\left[\vartheta\left(\mathfrak{d}\left(\mathfrak{v}_{n}^{i-1}, \mathfrak{v}_{n}^{i}\right)\right)\right]^{1-\varsigma_{2}} \\
& \leq \sum_{i=1}^{2 k+1}\left[\vartheta\left(\mathfrak{d}\left(\mathfrak{v}_{n-1}^{i-1}, \mathfrak{v}_{n-1}^{i}\right)\right)\right]^{\varsigma_{1}} .
\end{aligned}
$$

After simplification, we have

$$
\sum_{i=1}^{2 k+1}\left[\vartheta\left(\mathfrak{d}\left(\mathfrak{v}_{n}^{i-1}, \mathfrak{v}_{n}^{i}\right)\right)\right] \leq \sum_{i=1}^{2 k+1}\left[\vartheta\left(\mathfrak{d}\left(\mathfrak{v}_{n-1}^{i-1}, \mathfrak{v}_{n-1}^{i}\right)\right)\right]^{\left(\varsigma_{1} / 1-\varsigma_{2}\right)}
$$

By induction, it follows that for all $n \in \mathbb{N}$,

$$
\sum_{i=1}^{2 k+1}\left[\vartheta\left(\mathfrak{d}\left(\mathfrak{v}_{n}^{i-1}, \mathfrak{v}_{n}^{i}\right)\right)\right] \leq \sum_{i=1}^{2 k+1}\left[\vartheta\left(\mathfrak{d}\left(\mathfrak{v}_{0}^{i-1}, \mathfrak{v}_{0}^{i}\right)\right)\right]^{\left(\varsigma_{1} / 1-\varsigma_{2}\right)^{n}}
$$

which implies that

$$
\sum_{i=1}^{2 k+1}\left[\vartheta\left(\mathfrak{D}\left(\mathfrak{v}_{n}^{i-1}, \mathfrak{v}_{n}^{i}\right)\right)\right] \leq \sum_{i=1}^{2 k+1}\left[\vartheta\left(\mathfrak{d}\left(\mathfrak{v}_{0}^{i-1}, \mathfrak{v}_{0}^{i}\right)\right)\right]^{v^{n}} \text { for all } n \in \mathbb{N},
$$

where $v=\varsigma_{1} / 1-\varsigma_{2}<1$. Taking limit $n \longrightarrow \infty$ in the above inequality, we have

$$
\lim _{n \rightarrow \infty} \sum_{i=1}^{2 k+1} \vartheta\left(\mathfrak{d}\left(\mathfrak{v}_{n}^{i-1}, \mathfrak{v}_{n}^{i}\right)\right)=1
$$

which implies that

$$
\lim _{n \rightarrow \infty} \sum_{i=1}^{2 k+1} \mathfrak{d}\left(\mathfrak{v}_{n}^{i-1}, \mathfrak{v}_{n}^{i}\right)=0
$$

Since $\vartheta \in \Delta_{\vartheta}$ then there exist $0<Y<1$ and $0<\ell \leq \infty$, such that the following limit holds true:

$$
\lim _{n \rightarrow \infty} \sum_{i=1}^{2 k+1} \frac{\vartheta\left(\mathfrak{d}\left(\mathfrak{v}_{n}^{i-1}, \mathfrak{v}_{n}^{i}\right)\right)-1}{\left[\mathfrak{d}\left(\mathfrak{v}_{n}^{i-1}, \mathfrak{v}_{n}^{i}\right)\right]^{Y}}=\ell
$$

Assume that $\ell<\infty$ and $C=\ell / 2$. Thus, there exists $n_{0} \in \mathbb{N}$, such that

$$
\left|\sum_{i=1}^{2 k+1} \frac{\vartheta\left(\mathfrak{d}\left(\mathfrak{v}_{n}^{i-1}, \mathfrak{v}_{n}^{i}\right)\right)-1}{\left[\mathfrak{d}\left(\mathfrak{v}_{n}^{i-1}, \mathfrak{v}_{n}^{i}\right)\right]^{Y}}-\ell\right| \leq C, \text { for all } n \geq n_{0}
$$


Hence, we have

$$
\sum_{i=1}^{2 k+1} \frac{\vartheta\left(\mathfrak{d}\left(\mathfrak{v}_{n}^{i-1}, \mathfrak{v}_{n}^{i}\right)\right)-1}{\left[\mathfrak{d}\left(\mathfrak{v}_{n}^{i-1}, \mathfrak{v}_{n}^{i}\right)\right]^{Y}} \geq \ell-C=\frac{\ell}{2}=C \text {, for all } n \geq n_{0},
$$

and so we obtain

$$
n \sum_{i=1}^{2 k+1}\left[\mathfrak{d}\left(\mathfrak{v}_{n}^{i-1}, \mathfrak{v}_{n}^{i}\right)\right]^{Y} \leq \omega D\left[\sum_{i=1}^{2 k+1} \mathfrak{\vartheta}\left(\mathfrak{d}\left(\mathfrak{v}_{n}^{i-1}, \mathfrak{v}_{n}^{i}\right)\right)-1\right], \text { for all } n \geq n_{0},
$$

where $\mathfrak{d}=1 / C$. If $\ell=\infty$ then there exists $n_{0} \in \mathbb{N}$, such that

$$
\sum_{i=1}^{2 k+1} \frac{\mathfrak{9}\left(\mathfrak{d}\left(\mathfrak{v}_{n}^{i-1}, \mathfrak{v}_{n}^{i}\right)\right)-1}{\left[\mathfrak{d}\left(\mathfrak{v}_{n}^{i-1}, \mathfrak{v}_{n}^{i}\right)\right]^{Y}} \geq C, \text { for all } n \geq n_{0},
$$

which implies

$n \sum_{i=1}^{2 k+1}\left[\mathfrak{d}\left(\mathfrak{v}_{n}^{i-1}, \mathfrak{v}_{n}^{i}\right)\right]^{Y} \leq \omega D\left[\sum_{i=1}^{2 k+1} \vartheta\left(\mathfrak{d}\left(\mathfrak{p}_{n}^{i-1}, \mathfrak{v}_{n}^{i}\right)\right)-1\right]$, for all $n \geq n_{0}$,

where $\mathfrak{d}=1 / C$. Hence, in all cases, there exist $\mathfrak{d}>0$ and $n_{0} \in \mathbb{N}$, such that

$n \sum_{i=1}^{2 k+1}\left[\mathfrak{d}\left(\mathfrak{v}_{n}^{i-1}, \mathfrak{v}_{n}^{i}\right)\right]^{Y} \leq \omega D\left[\sum_{i=1}^{2 k+1} \mathfrak{\vartheta}\left(\mathfrak{d}\left(\mathfrak{v}_{n}^{i-1}, \mathfrak{v}_{n}^{i}\right)\right)-1\right]$, for all $n \geq n_{0}$

From inequalities (144) and (153), we have

$n \sum_{i=1}^{2 k+1}\left[\mathfrak{d}\left(\mathfrak{v}_{n}^{i-1}, \mathfrak{v}_{n}^{i}\right)\right]^{Y} \leq \omega D\left[\sum_{i=1}^{2 k+1}\left(\mathcal{(}\left(\mathfrak{d}\left(\mathfrak{v}_{0}^{i-1}, \mathfrak{v}_{0}^{i}\right)\right)\right)^{v^{n}}-1\right]$, for all $n \geq n_{0}$,

and taking limit as $n \longrightarrow \infty$ on both sides of the above inequality, we obtain

$$
n \sum_{i=1}^{2 k+1}\left[\mathfrak{d}\left(\mathfrak{v}_{n}^{i-1}, \mathfrak{v}_{n}^{i}\right)\right]^{Y}=0
$$

Thus, there exists $n_{0} \in \mathbb{N}$ such that

$$
\sum_{i=1}^{2 k+1} \mathfrak{d}\left(\mathfrak{v}_{n}^{i-1}, \mathfrak{v}_{n}^{i}\right) \leq \frac{1}{n^{1 / Y}}, \text { for all } n \geq n_{0} .
$$

By putting inequality (156) in inequality (138), we get

$$
\mathfrak{d}\left(q_{n}, q_{n+1}\right) \leq \frac{C}{n^{1 / Y}},
$$

where $C=1$.
Case 2. $N=2 k$ ( $N$ is even).

$$
\begin{aligned}
& \mathfrak{d}\left(q_{n}, q_{n+1}\right)=\mathfrak{d}\left(\mathfrak{v}_{n}^{0}, \mathbf{b}_{n}^{N}\right)=\mathfrak{d}\left(\mathfrak{v}_{n}^{0}, \mathfrak{v}_{n}^{2 k}\right) \leq \mathfrak{d}\left(\mathfrak{v}_{n}^{0}, \mathfrak{b}_{n}^{1}\right)+\mathfrak{d}\left(\mathfrak{v}_{n}^{1}, \mathfrak{v}_{n}^{2}\right) \\
& +\mathfrak{d}\left(\mathfrak{v}_{n}^{2}, \mathfrak{v}_{n}^{2 k}\right) \leq \mathfrak{d}\left(\mathfrak{v}_{n}^{0}, \mathfrak{v}_{n}^{1}\right)+\mathfrak{d}\left(\mathfrak{v}_{n}^{1}, \mathfrak{v}_{n}^{2}\right)+\cdots+\mathfrak{d}\left(\mathfrak{w}_{n}^{2 k-2}, \mathfrak{v}_{n}^{2 k}\right) \\
& =\sum_{i=1}^{2 k} \mathfrak{d}\left(\mathfrak{v}_{n}^{i-1}, \mathfrak{b}_{n}^{i}\right)-\mathfrak{d}\left(\mathfrak{b}_{n}^{2 k-2}, \mathfrak{v}_{n}^{2 k-1}\right)-\mathfrak{d}\left(\mathfrak{v}_{n}^{2 k-1}, \mathfrak{v}_{n}^{2 k}\right) \\
& +\mathfrak{d}\left(\mathfrak{v}_{n}^{2 k-2}, \mathfrak{v}_{n}^{2 k}\right) \leq \sum_{i=1}^{2 k} \mathfrak{d}\left(\mathfrak{p}_{n}^{i-1}, \mathfrak{v}_{n}^{i}\right)+\mathfrak{d}\left(\mathfrak{v}_{n}^{2 k-2}, \mathfrak{v}_{n}^{2 k}\right) .
\end{aligned}
$$

By the same arguments used in Case 1, we deduce that

$$
\sum_{i=1}^{2 k} \mathfrak{D}\left(\mathfrak{v}_{n}^{i-1}, \mathfrak{v}_{n}^{i}\right) \leq \frac{1}{n^{1 / Y}}, \text { for all } n \geq n_{0} .
$$

Indeed, from (136), we have

$$
\mathfrak{D}\left(\mathfrak{v}_{n}^{2 k-2}, \mathfrak{F}_{\mathfrak{v}^{2 k-1}}^{2 k-2}\right)=\mathfrak{d}\left(\mathfrak{v}_{n}^{2 k}, \mathfrak{F}_{n-1}^{2 k}\right)=\mathfrak{d}(\mathscr{Q}, \mathscr{P}) .
$$

Since $\mathfrak{F}$ is $(\vartheta, \mathfrak{g})$-proximal contraction, we have $\mathfrak{\vartheta}\left(\mathfrak{d}\left(\mathfrak{F}_{n-1}^{2 k-2}, \mathfrak{F}_{n-1}^{2 k}\right)\right) \leq\left[\mathfrak{\vartheta}\left(\mathfrak{d}\left(\mathfrak{p}_{n-1}^{2 k-2}, \mathfrak{v}_{n-1}^{2 k}\right)\right)\right]^{\varsigma_{1}}\left[\mathfrak{\vartheta}\left(\mathfrak{d}\left(\mathfrak{p}_{n}^{2 k-2}, \mathfrak{v}_{n}^{2 k}\right)\right)\right]^{\varsigma_{2}}$

The pair $(\mathscr{Q}, \mathscr{P})$ satisfies weak $P$-property; then, we can write

$$
\mathfrak{d}\left(\mathfrak{v}_{n}^{2 k-2}, \mathfrak{v}_{n}^{2 k}\right) \leq \mathfrak{d}\left(\mathfrak{F}_{n-1}^{2 k-2}, \mathfrak{F}_{n-1}^{2 k}\right)
$$

Since $\vartheta$ is increasing then the above inequality becomes

$$
\mathfrak{\vartheta}\left(\mathfrak{d}\left(\mathfrak{v}_{n}^{2 k-2}, \mathfrak{v}_{n}^{2 k}\right)\right) \leq \mathfrak{\vartheta}\left(\mathfrak{d}\left(\mathfrak{F}_{n-1}^{2 k-2}, \mathfrak{F}_{n-1}^{2 k}\right)\right) .
$$

Then, from inequalities (161) and (163), we have

$$
\vartheta\left(\mathfrak{d}\left(\mathfrak{w}_{n}^{2 k-2}, \mathfrak{v}_{n}^{2 k}\right)\right) \leq\left[\vartheta\left(\mathfrak{d}\left(\mathfrak{v}_{n-1}^{2 k-2}, \mathfrak{v}_{n-1}^{2 k}\right)\right)\right]^{\mathcal{\zeta}_{1}}\left[\vartheta\left(\mathfrak{d}\left(\mathfrak{v}_{n}^{2 k-2}, \mathfrak{v}_{n}^{2 k}\right)\right)\right]^{\varsigma_{2}}
$$

After further simplifications,

$$
\left.\vartheta\left(\mathfrak{d}\left(\mathfrak{v}_{n}^{2 k-2}, \mathfrak{v}_{n}^{2 k}\right)\right)\right]^{1-\varsigma_{2}} \leq\left[\mathfrak{\vartheta}\left(\mathfrak{d}\left(\mathfrak{v}_{n-1}^{2 k-2}, \mathfrak{v}_{n-1}^{2 k}\right)\right)\right]^{\varsigma_{1}} .
$$

By induction, it follows that for all $n \in \mathbb{N}$,

$$
\left.\left.\vartheta\left(\mathfrak{d}\left(\mathfrak{v}_{n}^{2 k-2}, \mathfrak{v}_{n}^{2 k}\right)\right)\right] \leq \vartheta\left(\mathfrak{d}\left(\mathfrak{v}_{0}^{2 k-2}, \mathfrak{v}_{0}^{2 k}\right)\right)\right]^{\left(\varsigma_{1} / 1-\varsigma_{2}\right)^{n}}, \text { for all } n \in \mathbb{N},
$$

where $v=\varsigma_{1} / 1-\varsigma_{2}$. Taking the limit $n \longrightarrow \infty$ in above 
inequality, we have

$$
\lim _{n \longrightarrow \infty}\left[\vartheta\left(\mathfrak{d}\left(\mathfrak{b}_{n}^{2 k-2}, \mathfrak{v}_{n}^{2 k}\right)\right)\right]=1 .
$$

Similarly, from condition $\left(\vartheta_{2}\right)$, we have

$$
\lim _{n \longrightarrow \infty} \mathfrak{d}\left(\mathfrak{v}_{n}^{2 k-2}, \mathfrak{v}_{n}^{2 k}\right)=0 .
$$
that

Similarly, from condition $\left(\vartheta_{3}\right)$, there exists $n_{1} \in \mathbb{N}$, such

$$
\mathfrak{d}\left(\mathfrak{v}_{n}^{2 k-2}, \mathfrak{v}_{n}^{2 k}\right) \leq \frac{1}{n^{1 / Y}}, \text { for all } n \geq n_{1} .
$$

Then, from inequalities (158), (159), and (169), we have

$$
\mathfrak{D}\left(q_{n}, q_{n+1}\right) \leq \frac{C}{n^{1 / Y}}, \text { for all } n \geq n_{1} .
$$

where $C=2$. Let us prove that $\left\{q_{n}\right\}$ is a Cauchy sequence. Let $n, m \in N$ such that $m>n$. We suppose that $m$ is odd $(m=2 k+1)$ since the case $m=2 k$ is similar. Note that $q_{n}=$ $\mathfrak{v}_{0}^{n}, q_{n+1}=\mathfrak{v}_{n}^{N}$, and $\mathfrak{v}_{0}^{n} \neq \mathfrak{v}_{n}^{N}$ for all $n$ since the path $\left(\mathfrak{v}_{n}^{i}\right)_{i=0,1, \cdots, N}$ is elementary. Then, by using the rectangular metric, we obtain

$$
\begin{aligned}
\mathfrak{d}\left(q_{n}, q_{m}\right) \leq & \mathfrak{d}\left(q_{n}, q_{n+1}\right)+\mathfrak{d}\left(q_{n+1}, q_{n+2}\right) \\
& +\mathfrak{d}\left(q_{n+2}, q_{n+3}\right)+\cdots+\mathfrak{d}\left(q_{m-1}, q_{m}\right) \\
\leq & \frac{C}{n^{1 / Y}}+\frac{C}{(n+1)^{1 / Y}}+\frac{C}{(n+2)^{1 / Y}}+\cdots \\
& +\frac{C}{(n+m-2)^{1 / Y}} \leq \sum_{i=n}^{\infty} \frac{C}{i^{1 / Y}},
\end{aligned}
$$

for all $m \geq n \geq N$, where $N=\max \left\{n_{0}, n_{1}\right\}$. Hence, we obtain

$$
\mathfrak{D}\left(q_{n}, q_{m}\right) \leq \sum_{i=n}^{\infty} \frac{C}{i^{1 / Y}}, \text { for all } n \geq N, m \in \mathbb{N}
$$

We deduce that $\left\{q_{n}\right\}$ is a Cauchy sequence in $Q_{0} \subseteq \mathscr{Q} \subset \mathscr{X}$ . By using the completeness of Branciari metric space $\mathscr{X}$ and closeness of $\mathbb{Q}_{0}$, there exists $q^{*} \in \mathbb{Q}_{0}$ such that $q_{n} \longrightarrow q^{*} \in \mathbb{Q}_{0}$. Since $q^{*} \in \mathbb{Q}_{0}, \mathfrak{\Im}\left(\mathbb{Q}_{0}\right) \subseteq \mathscr{P}_{0}$ then $\mathfrak{\Im} q^{*} \in \mathscr{P}_{0}$, thus there exists a point $z \in \mathbb{Q}_{0}$ such that $z=q^{*}$ and

$$
\mathfrak{d}\left(z, \mathfrak{F} q^{*}\right)=\mathfrak{d}(\mathscr{Q}, \mathscr{P}) .
$$

As $\mathfrak{F}$ is $(\mathcal{\vartheta}, \mathfrak{g})$-proximal contraction, we obtain

$$
\mathfrak{\vartheta}\left(\mathfrak{d}\left(\mathfrak{J} q^{*}, \mathfrak{\mho} q_{n}\right)\right) \leq\left[\mathfrak{\vartheta}\left(\mathfrak{d}\left(q^{*}, q_{n}\right)\right)\right]^{\varsigma_{1}}\left[\mathfrak{V}\left(\mathfrak{d}\left(q_{n+1}, z\right)\right)\right]^{\varsigma_{2}} .
$$

Then, by using the weak $P$-property of subsets $(\mathscr{Q}, \mathscr{P})$ of Branciari metric space,we have

$$
\vartheta\left(\mathfrak{D}\left(z, q_{n+1}\right)\right) \leq \mathfrak{\vartheta}\left(\mathfrak{d}\left(\mathfrak{F} q^{*}, \mathfrak{\mho} q_{n}\right)\right) .
$$

Hence, we have

$$
\mathfrak{\vartheta}\left(\mathfrak{d}\left(z, q_{n+1}\right)\right) \leq\left[\vartheta\left(\mathfrak{d}\left(q^{*}, q_{n}\right)\right)\right]^{\varsigma_{1}}\left[\vartheta\left(\mathfrak{d}\left(q_{n+1}, z\right)\right)\right]^{\varsigma_{2}} .
$$

Further,

$$
\left[\mathcal{Y}\left(\mathfrak{d}\left(q_{n+1}, z\right)\right)\right]^{1-\varsigma_{2}} \leq\left[\mathcal{Y}\left(\mathfrak{D}\left(q^{*}, q_{n}\right)\right)\right]^{\varsigma_{1}}<\left[\mathcal{Y}\left(\mathfrak{D}\left(q^{*}, q_{n}\right)\right)\right]^{1-\varsigma_{2}} .
$$

This implies that

$$
\left[\vartheta\left(\mathfrak{D}\left(q_{n+1}, z\right)\right)\right]<\left[\vartheta\left(\mathfrak{D}\left(q^{*}, q_{n}\right)\right)\right] .
$$

As 9 is increasing, we have

$$
\mathfrak{d}\left(q_{n+1}, z\right)<\mathfrak{d}\left(q^{*}, q_{n}\right)
$$

Then, by rectangular property and using above inequality and inequality (170), we have

$$
\begin{aligned}
\mathfrak{d}\left(q^{*}, z\right) & \leq \mathfrak{d}\left(q^{*}, q_{n}\right)+\mathfrak{d}\left(q_{n}, q_{n+1}\right)+\mathfrak{d}\left(q_{n+1}, z\right) \\
& <\mathfrak{d}\left(q^{*}, q_{n}\right)+\frac{C}{n^{1 / Y}}+\mathfrak{d}\left(q^{*}, q_{n}\right) .
\end{aligned}
$$

Now, by taking limit as $n \longrightarrow \infty$, we have $z=q^{*}$.

$$
\mathfrak{d}\left(\mathfrak{g} q^{*}, \mathfrak{\mho} q^{*}\right)=\mathfrak{d}(\mathscr{Q}, \mathscr{P}) .
$$

Thus, $q^{*}$ is a coincidence best proximity point of pair of mappings $(\mathfrak{F}, \mathfrak{g})$.

Uniqueness. We now show that $q^{*}$ is a unique best proximity point of $\mathfrak{F}$. Let $w$ be another best proximity point of $\mathfrak{F}$ with $q^{*}=w$. Hence,

$$
\mathfrak{D}(\mathscr{Q}, \mathscr{P})=\mathfrak{d}(w, \mathfrak{F} w) .
$$

Then, by using properties of $\mathfrak{F}$ and $\mathfrak{g}$ and reasoning as above, we obtain that

$$
\begin{aligned}
{\left[\mathcal{\vartheta}\left(\mathfrak{d}\left(q^{*}, w\right)\right)\right] } & \leq \mathfrak{V}\left(\mathfrak{d}\left(\mathfrak{\mho} q^{*}, \mathfrak{J} w\right)\right) \leq\left[\vartheta\left(\mathfrak{D}\left(q^{*}, w\right)\right)\right]^{\varsigma_{1}}\left[\mathcal{V}\left(\mathfrak{d}\left(q^{*}, w\right)\right)\right]^{\varsigma_{2}} \\
& \leq\left[\mathfrak{\vartheta}\left(\mathfrak{d}\left(q^{*}, w\right)\right)\right]^{\varsigma_{1}+\varsigma_{2}}<\left[\mathcal{V}\left(\mathfrak{d}\left(q^{*}, w\right)\right)\right],
\end{aligned}
$$

which is a contradiction. Therefore, $q^{*}=w$. Hence, $\mathfrak{F}$ has a unique best proximity point.

Corollary 45. Let $\mathfrak{F}: \mathbb{Q} \longrightarrow \mathscr{P}$ be a $(\mathfrak{\vartheta}, \mathfrak{g})$-generalized proximal contraction. Moreover, $\mathbb{Q}_{0}$ is a nonempty closed subset in Branciari metric space $\mathscr{X}$ and $\mathfrak{F}\left(\mathscr{Q}_{0}\right) \subseteq \mathscr{P}_{0}$. If there exists a path $\left(z^{i}\right)_{i=0}^{\mathfrak{b}} \subseteq Q_{0}$ in $\mathfrak{g}$ between two elements $q$ and $p$ then there exists a unique best proximity of $\mathfrak{F}$ provided that there exists $q_{0}, q_{1} \in \mathbb{Q}_{0}$ such that there is an elementary path in $\mathbb{Q}_{0}$ between them and

$$
\mathfrak{d}\left(q_{1}, \mathfrak{\mho} q_{0}\right)=\mathfrak{d}(\mathscr{Q}, \mathscr{P}) .
$$

Proof. Let $q_{0}, q_{1} \in \mathbb{Q}_{0}$ such that $\mathfrak{d}\left(q_{1}, \mathfrak{F} q_{0}\right)=\mathfrak{d}(\mathscr{Q}, \mathscr{P})$. A path 
$\left(\mathfrak{v}_{0}^{i}\right)_{i=0}^{N}$ in $\mathfrak{g}$ is a sequence containing points of $\mathbb{Q}_{0}$. Following arguments similar to those given in the proof of Corollary 44, we obtain

$$
\begin{aligned}
\mathfrak{d}\left(\mathfrak{v}_{n}^{i-1}, \mathfrak{F}_{n-1}^{i-1}\right) & =\mathfrak{d}(\mathscr{Q}, \mathscr{P})=\mathfrak{D}\left(\mathfrak{v}_{n}^{i}, \mathfrak{F}_{n-1}^{i}\right), \text { for all } i \\
& =1,2, \cdots, N, n \in \mathbb{N} \cup\{0\} .
\end{aligned}
$$
have

Since $\mathfrak{F}$ is $(\mathfrak{\vartheta}, \mathfrak{g})$-generalized proximal contraction, we

$$
\vartheta\left(\mathfrak{D}\left(\mathfrak{v}_{n}^{i-1}, \mathfrak{v}_{n}^{i}\right)\right) \leq\left[\vartheta\left(\mathfrak{D}\left(\mathfrak{v}_{n-1}^{i-1}, \mathfrak{v}_{n-1}^{i}\right)\right)\right]^{\varsigma_{1}}\left[\vartheta\left(\mathfrak{d}\left(\mathfrak{v}_{n}^{i}, \mathfrak{v}_{n}^{i-1}\right)\right)\right]^{\varsigma_{2}}
$$

Again by using the arguments similar to those given in the proof of Corollary 44, we have

$$
\mathfrak{d}\left(q_{n}, q_{n+1}\right) \leq \frac{C}{n^{1 / Y}}, \text { for all } n \geq n_{0}
$$

where $C=1$. Again by using the arguments similar to those given in the proof of Corollary 44 and since $\mathfrak{F}$ is $(\vartheta, \mathfrak{g})$ -proximal contraction, we have

$$
\begin{gathered}
\vartheta\left(\mathfrak{d}\left(\mathfrak{v}_{n}^{2 k-2}, \mathfrak{v}_{n}^{2 k}\right)\right) \leq\left[\vartheta\left(\mathfrak{d}\left(\mathfrak{v}_{n-1}^{2 k-2}, \mathfrak{v}_{n-1}^{2 k}\right)\right)\right]^{\varsigma_{1}}\left[\vartheta\left(\mathfrak{d}\left(\mathfrak{v}_{n}^{2 k}, \mathfrak{v}_{n}^{2 k-2}\right)\right)\right]^{\varsigma_{2}} \\
\cdot\left[\vartheta\left(\mathfrak{d}\left(\mathfrak{v}_{n}^{2 k-2}, \mathfrak{v}_{\mathrm{n}}^{2 k}\right)\right)\right]^{1-\varsigma_{2}} \leq\left[\vartheta\left(\mathfrak{d}\left(\mathfrak{v}_{n-1}^{2 k-2}, \mathfrak{v}_{n-1}^{2 k}\right)\right)\right]^{\varsigma_{1}} .
\end{gathered}
$$

By using the arguments similar to those given in the proof of Corollary 44, we deduce that $\left\{q_{n}\right\}$ is a Cauchy

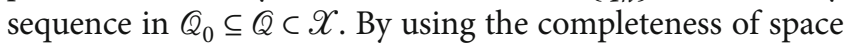
$\mathscr{X}$ and $\mathscr{Q}_{0}$ is closed, there exists $q^{*} \in \mathscr{Q}_{0}$ such that $q_{n} \longrightarrow q^{*}$ $\in \mathscr{Q}_{0}$. Since $q^{*} \in \mathbb{Q}_{0}, \mathfrak{F}\left(\mathscr{Q}_{0}\right) \subseteq \mathscr{P}_{0}$ then $\mathfrak{F} q^{*} \in \mathscr{P}_{0}$; thus, there exists a point $z \in \mathbb{Q}_{0}$ such that $z=q^{*}$ and

$$
\mathfrak{d}\left(z, \mathfrak{F} q^{*}\right)=\mathfrak{d}(\mathscr{Q}, \mathscr{P})
$$

As $\mathfrak{F}$ is $(\vartheta, \mathfrak{g})$-generalized proximal contraction, we have

$$
\begin{aligned}
\vartheta\left(\mathfrak{d}\left(z, q_{n+1}\right)\right) & \leq\left[\vartheta\left(\mathfrak{d}\left(q^{*}, q_{n}\right)\right)\right]^{\varsigma_{1}}\left[\vartheta\left(\mathfrak{d}\left(q_{n+1}, z\right)\right)\right]^{\varsigma_{2}}\left[\vartheta\left(\mathfrak{d}\left(z, q_{n+1}\right)\right)\right]^{1-\varsigma_{2}} \\
& \leq\left[\vartheta\left(\mathfrak{d}\left(q^{*}, q_{n}\right)\right)\right]^{\varsigma_{1}}<\left[\vartheta\left(\mathfrak{d}\left(q^{*}, q_{n}\right)\right)\right]^{1-\varsigma_{2}} .
\end{aligned}
$$

Following arguments similar to those given in the proof of Corollary 44 , we obtain that $q^{*}$ is the best proximity point of the mapping $\mathfrak{F}$.

Uniqueness. Now we have to show that $q^{*}$ is a unique best proximity point of $\mathfrak{F}$. Let $w$ be another best proximity point of $\mathfrak{F}$ with $q^{*}=w$. Hence,

$$
\mathfrak{d}(\mathfrak{Q}, \mathscr{P})=\mathfrak{d}(w, \mathfrak{F} w)
$$

Then, by using properties of $\mathfrak{F}$ and $\mathfrak{g}$ and reasoning as above, we obtain that

$$
\begin{aligned}
{\left[\vartheta\left(\mathfrak{d}\left(q^{*}, w\right)\right)\right] } & \leq\left[\vartheta\left(\mathfrak{d}\left(q^{*}, w\right)\right)\right]^{\varsigma_{1}}\left[\vartheta\left(\mathfrak{d}\left(q^{*}, w\right)\right)\right]^{\varsigma_{2}} \\
& \leq\left[\vartheta\left(\mathfrak{d}\left(q^{*}, w\right)\right)\right]^{\varsigma_{1}+\varsigma_{2}}<\left[\vartheta\left(\mathfrak{d}\left(q^{*}, w\right)\right)\right],
\end{aligned}
$$

which is a contradiction. Therefore, $q^{*}=w$. Hence, $\mathfrak{F}$ has a unique best proximity point.

\section{Conclusion}

In this paper, we define $\left(\vartheta, \alpha^{+}, \mathfrak{g}\right)$-proximal contraction and provide the existence results for coincidence best proximity point in Branciari metric space. The important aspect of Branciari metric space is that it is not continuous; we dealt with discontinuity of Branciari metric space and obtained the desired results. As an application, we derive the coincidence point and fixed point results for some self mappings. We also introduce the notion of $(\vartheta, \mathfrak{g})$-proximal contraction and provide an application to graph theory in the setting of Branciari metric space. Some examples are also provided to illustrate the novelty of the result proved herein.

\section{Data Availability}

No data were used to support this study.

\section{Conflicts of Interest}

The authors declare that they have no competing interests.

\section{Authors' Contributions}

All authors contributed equally and significantly in writing this paper. All authors have read and agreed to the published version of the manuscript.

\section{Acknowledgments}

We appreciate the reviewer's careful reading and remarks which helped us to improve the paper. The authors are grateful to the Basque Government for Grant IT1207-19.

\section{References}

[1] S. Banach, "Sur les opérations dans les ensembles abstraits et leur application aux équations intégrales," Fundamenta Mathematicae, vol. 3, pp. 133-181, 1922.

[2] K. Fan, "Extensions of two fixed point theorems of F. E. Browder," Mathematische Zeitschrift, vol. 112, no. 3, pp. 234-240, 1969.

[3] N. Hussain, M. Hezarjaribi, M. A. Kutbi, and P. Salimi, "Best proximity results for Suzuki and convex type contractions," Fixed Point Theory and Applications, vol. 2016, no. 1, 2016.

[4] M. Jleli and B. Samet, "A new generalization of the Banach contraction principle," Journal of Inequalities and Applications, vol. 2014, Article ID 38, 2014.

[5] P. Kumam and A. F. R. L. de Hierro, "On existence and uniqueness of g-best proximity points under $(\phi, \theta, \alpha, \mathfrak{g})$-contractivity conditions and consequences," Abstract and Applied Analysis, vol. 2014, Article ID 234027, 14 pages, 2014. 
[6] A. Latif, N. Saleem, and M. Abbas, "Alpha-optimal best proximity point result involving proximal contraction mappings in fuzzy metric spaces," Journal of Nonlinear Sciences and Applications, vol. 10, no. 1, pp. 92-103, 2017.

[7] M. De La Sen, M. Abbas, and N. Saleem, "On optimal fuzzy best proximity coincidence points of proximal contractions involving cyclic mappings in non-Archimedean fuzzy metric spaces," Mathematics, vol. 5, no. 2, p. 22, 2017.

[8] Z. Raza, N. Saleem, and N. Abbas, "Optimal coincidence points of proximal quasi-contraction mappings in nonArchimedean fuzzy metric spaces," Journal of Nonlinear Sciences and Applications, vol. 9, no. 6, pp. 3787-3801, 2016.

[9] N. Saleem, M. Abbas, and Z. Raza, "Optimal coincidence best approximation solution in non-Archimedean fuzzy metric spaces," Iranian Journal of Fuzzy Systems, vol. 13, no. 3, pp. 113-124, 2016.

[10] M. Abbas, N. Saleem, and M. De la Sen, "Optimal coincidence point results in partially ordered non-Archimedean fuzzy metric spaces," Fixed Point Theory and Applications, vol. 1, no. 1, 2016.

[11] N. Saleem, B. Ali, M. Abbas, and Z. Raza, "Fixed points of Suzuki type generalized multivalued mappings in fuzzy metric spaces with applications," Fixed Point Theory and Applications, vol. 2015, no. 1, 2015.

[12] N. Saleem, I. Habib, and M. D. Sen, "Some new results on coincidence points for multivalued Suzuki-type mappings in fairly complete spaces," Computation, vol. 8, no. 1, p. 17, 2020.

[13] V. Sankar Raj, "A best proximity point theorem for weakly contractive non-self mappings," Nonlinear Analysis, vol. 74, no. 14, pp. 4804-4808, 2011.

[14] J. Zhang, Y. Su, and Q. Cheng, "A note on 'A best proximity point theorem for Geraghty-contractions', 'Fixed Point Theory and Applications, vol. 2013, 2013.

[15] A. Branciari, "A fixed point theorem of Banach-Caccioppoli type on a class of generalized metric spaces," Publicationes Mathematicae Debrecen, vol. 76, no. 4, pp. 493-494, 2010.

[16] Z. Kadelburg and S. Radenovic, "On generalized metric spaces: a survey," TWMS Journal of Pure and Applied Mathematics, vol. 5, pp. 3-13, 2014.

[17] I. R. Sarma, J. M. Rao, and S. S. Rao, "Contractions over generalized metric spaces," Journal of Nonlinear Sciences and Applications, vol. 2, no. 3, pp. 180-182, 2009.

[18] W. A. Kirk and N. Shahzad, "Generalized metrics and Caristi's theorem," Fixed Point Theory and Applications, vol. 2013, no. $1,2013$.

[19] M. Gableh, "Global optimal solutions of nonself mappings," UPB Scientific Bulletin, Series A, vol. 75, no. 3, 2013.

[20] F. S. Beckman and D. A. Quarles, "On isometries of Euclidean spaces," Proceedings of the American Mathematical Society, vol. 4, no. 5, pp. 810-815, 1953.

[21] R. K. Williams, "Expansive mappings," The American Mathematical Monthly, vol. 75, no. 5, pp. 522-523, 1968.

[22] N. Saleem, M. Abbas, B. Bin-Mohsin, and S. Radenovic, "Pata type best proximity point results in metric spaces," Miskolc Mathematical Notes, vol. 21, no. 1, pp. 367-386, 2020.

[23] M. Gableh, "Best proximity point theorems via proximal nonself mappings," Journal of Optimization Theory and Applications, vol. 164, no. 2, pp. 565-576, 2015.

[24] J. Jachymski, "The contraction principle for mappings on a metric space with a graph," Proceedings of American Mathematical Society, vol. 136, pp. 1359-1373, 2008. 